\title{
ZETA FUNCTIONS, HEAT KERNELS, AND SPECTRAL ASYMPTOTICS ON DEGENERATING FAMILIES OF DISCRETE TORI
}

\author{
GAUTAM CHINTA, JAY JORGENSON, \\ AND ANDERS KARLSSON
}

\begin{abstract}
By a discrete torus we mean the Cayley graph associated to a finite product of finite cycle groups with the generating set given by choosing a generator for each cyclic factor. In this article we examine the spectral theory of the combinatorial Laplacian for sequences of discrete tori when the orders of the cyclic factors tend to infinity at comparable rates. First, we show that the sequence of heat kernels corresponding to the degenerating family converges, after rescaling, to the heat kernel on an associated real torus. We then establish an asymptotic expansion, in the degeneration parameter, of the determinant of the combinatorial Laplacian. The zeta-regularized determinant of the Laplacian of the limiting real torus appears as the constant term in this expansion. On the other hand, using a classical theorem by Kirchhoff, the determinant of the combinatorial Laplacian of a finite graph divided by the number of vertices equals the number of spanning trees, called the complexity, of the graph. As a result, we establish a precise connection between the complexity of the Cayley graphs of finite abelian groups and heights of real tori. It is also known that spectral determinants on discrete tori can be expressed using trigonometric functions and that spectral determinants on real tori can be expressed using modular forms on general linear groups. Another interpretation of our analysis is thus to establish a link between limiting values of certain products of trigonometric functions and modular forms. The heat kernel analysis which we employ uses a careful study of $I$-Bessel functions. Our methods extend to prove the asymptotic behavior of other spectral invariants through degeneration, such as special values of spectral zeta functions and Epstein-Hurwitz-type zeta functions.
\end{abstract}

Received January 30, 2009. Accepted November 3, 2009.

The first and second authors acknowledge support provided by grants from the National Science Foundation and the Professional Staff Congress of the City University of New York. The third author thanks the Swedish Research Council, the Göran Gustafsson Foundation, and the G. S. Magnussons fund for support. 


\section{$\S 1$. Introduction}

\subsection{Historical Review}

The problems we study in the present article begin with the following very elementary question. For any $d \geq 1$, let $N=\left(n_{1}, \ldots, n_{d}\right)$ denote a $d$-tuple of positive integers, and consider the product

$$
D(N)=\prod_{K \neq 0}\left(2 d-2 \cos \left(2 \pi k_{1} / n_{1}\right)-\cdots-2 \cos \left(2 \pi k_{d} / n_{d}\right)\right),
$$

where the product is over all $d$-tuples $K=\left(k_{1}, \ldots, k_{d}\right)$ of nonnegative integers with $k_{j}<n_{j}$, omitting the zero vector in the product. The basic question is the following: What is the asymptotic behavior of $D(N)$ as $N \rightarrow$ $\infty$ ? Setting $V(N)=n_{1}, \ldots, n_{d},(\log D(N)) / V(N)$ can be interpreted as an improper Riemann sum, and we have the limiting formula

$$
\begin{aligned}
& \frac{1}{V(N)} \log D(N) \\
& \quad \rightarrow \int_{\mathbf{Z}^{d} \backslash \mathbf{R}^{d}} \log \left(2 d-2 \cos \left(2 \pi x_{1}\right)-\cdots-2 \cos \left(2 \pi x_{d}\right)\right) d x_{1} \cdots d x_{d}
\end{aligned}
$$

as each $n_{j} \rightarrow \infty$. The integral in (2) exists in the sense of improper integrals from elementary calculus, and the convergence of $D(N) / V(N)$ to the improper integral in (2) can be verified easily using the monotonicity of $\log x$ for $x>0$ and calculus.

One can view $D(N)$ as a determinant of a naturally defined matrix from graph theory. Quite generally, associated to any finite graph is a discrete Laplacian which acts on the finite-dimensional space of complex-valued functions whose domain of definition is the space of vertices of the graph. With our normalization of the Laplacian, defined in Section 2.4 below, $D(N)$ is equal to the product of the nonzero eigenvalues of the Laplacian associated to a graph which we call a discrete torus.

The study of $D(N)$ takes on an entirely new level of significance beginning with the 1847 paper of Kirchhoff [18], which further recognizes $D(N)$ as a fundamental invariant from graph theory. A spanning tree of a graph is a subgraph, which contains precisely one path between each pair of vertices of the original graph. Kirchhoff's theorem states that the number of spanning trees is equal to $D(N) / V(N)$, the product of the nonzero eigenvalues of the Laplacian divided by the number of vertices. 
Modern mathematics, theoretical computer science, and statistical physics contain numerous studies which in some way involve the number of spanning trees of a given graph, determinants of Laplacians, or other symmetric functions of eigenvalues which form basic invariants. With all this, we see that the invariant $D(N)$ has considerable significance far beyond the elementary considerations which allow for its definition.

\subsection{Summary of the main results}

Our work begins with the results from [15], which establishes a theta inversion formula for the discrete Laplacian acting on the space of vertices of $n \mathbf{Z} \backslash \mathbf{Z}$. Let $I_{x}(t)$ be the classical $I$-Bessel function, reviewed in detail in Section 2.1 below. Then, using the general concept of theta functions with inversion formulas constructed from heat kernels (see, e.g., [14]), the authors in [15] prove that for any $t>0$ and integer $x$, the following identity holds for the theta function associated to the discrete torus $n \mathbf{Z} \backslash \mathbf{Z}$ :

$$
\theta_{n}(t, x)=\frac{1}{n} \sum_{k=0}^{n-1} e^{-(2-2 \cos (2 \pi k / n)) t+2 \pi i k x / n}=e^{-2 t} \sum_{j=-\infty}^{\infty} I_{x+j n}(2 t)
$$

where $I_{x}(t)$ denotes the $I$-Bessel function (see Section 2.2). The generalization of $(3)$ to the $d$-dimensional discrete torus, defined as the product space

$$
D T_{N}=\prod_{j=1}^{d} n_{j} \mathbf{Z} \backslash \mathbf{Z}
$$

comes from taking a $d$-fold product of the theta functions in (3). By computing an integral transform of the $d$-dimensional theta inversion, we obtain the following formula, given in Theorem 3.6. For any $s \in \mathbf{C}$ with $\operatorname{Re}\left(s^{2}\right)>0$, we have that

$$
\sum_{\Lambda_{j} \neq 0} \log \left(s^{2}+\Lambda_{j}\right)=V(N) \mathcal{I}_{d}(s)+\mathcal{H}_{N}(s)
$$

where

$$
\begin{aligned}
& \left\{\Lambda_{j}\right\}=\left\{2 d-2 \cos \left(2 \pi k_{1} / n_{1}\right)-\cdots-2 \cos \left(2 \pi k_{d} / n_{d}\right)\right\}, \\
& \text { with } k_{j}=0, \ldots, n_{j}-1
\end{aligned}
$$

as in (1);

$$
\mathcal{I}_{d}(s)=\int_{0}^{\infty}\left(e^{-2 d t} e^{-s^{2} t} I_{0}(2 t)^{d}-e^{-t}\right) \frac{d t}{t}
$$


and

$$
\mathcal{H}_{N}(s)=-\int_{0}^{\infty}\left(e^{-s^{2} t}\left[\theta_{N}(t)-V(N) e^{-2 d t} I_{0}(2 t)^{d}-1\right]+e^{-t}\right) \frac{d t}{t} .
$$

We now consider a sequence of integral vectors $N(u)=\left(n_{1}(u), \ldots, n_{d}(u)\right)$ parametrized by $u \in \mathbf{Z}$ such that $n_{j}(u) / u \rightarrow \alpha_{j}$ as $u \rightarrow \infty$ for each $j$. Let $A$ be the diagonal matrix with the numbers $\alpha_{i}$ on the diagonal, and let $V(A)=$ $\alpha_{1}, \ldots, \alpha_{d} \neq 0$. Through a careful study of the infinite series of $I$-Bessel functions in (3), we obtain the following theorem, which is one of the main results of the present article.

Main TheOREM. Let $\log \operatorname{det}^{*} \boldsymbol{\Delta}_{D T, N(u)}$ be the log-determinant of the Laplacian of nonzero eigenvalues on the d-dimensional discrete torus associated to $N(u)$, and let $\log \operatorname{det}^{*} \boldsymbol{\Delta}_{\mathrm{RT}, A}$ be the log-determinant of the Laplacian on the real torus $A \mathbf{Z}^{d} \backslash \mathbf{R}^{d}$. Then

$$
\log \operatorname{det}^{*} \Delta_{D T, N(u)}=V(N(u)) \mathcal{I}_{d}(0)
$$

$$
+\log u^{2}+\log \operatorname{det}^{*} \boldsymbol{\Delta}_{\mathrm{RT}, A}+o(1), \quad \text { as } u \rightarrow \infty,
$$

where

$$
\mathcal{I}_{d}(0)=\left(\log 2 d-\int_{0}^{\infty}\left(e^{-2 d t}\left(I_{0}(2 t)^{d}-1\right) \frac{d t}{t}\right) .\right.
$$

A number of facets of (5) are interesting. First, the determinant $\operatorname{det}^{*} \boldsymbol{\Delta}_{D T, N(u)}$ is a legitimate finite product of eigenvalues, whereas $\operatorname{det}^{*} \boldsymbol{\Delta}_{\mathrm{RT}, A}$ is defined through zeta function regularization. From this point of view, we have connected a zeta-regularized determinant with a classical determinant. Moreover, since the first terms in (5) are universal, this allows for the possibility of transferring knowledge, for example, from the minimal spectral determinants for real tori, as studied in [5] and [23], to minimal spectral determinants for discrete tori or, alternatively, the minimal number of spanning trees. In this context it is relevant to remark that the main theorem holds without any changes for general tori (see Section 7.5). Second, the discrete torus $D T_{N}$ can be viewed as a lattice on the real torus $A \mathbf{Z}^{d} \backslash \mathbf{R}^{d}$, and the degeneration which occurs when $u$ tends to infinity amounts to considering a family of discrete tori which are becoming uniformly dense in $A \mathbf{Z}^{d} \backslash \mathbf{R}^{d}$. The main theorem above proves a type of "rescaled continuity" when studying the asymptotic behavior of spectral determinants. Finally, the classical Kronecker limit formula for Epstein zeta functions amounts to 
the evaluation of $\log \operatorname{det}^{*} \boldsymbol{\Delta}_{\mathrm{RT}, A}$ in terms of a generalization of Dedekind's eta function to a $\mathrm{GL}(d, \mathbf{Z})$ modular form. With this, we have established a precise connection between the asymptotics of the number of spanning trees on families of discrete tori and modular forms.

The verification of the main theorem in the case $d=1$ can be carried out directly; the details are presented in Section 6.1. When $d=2$, additional explicit computations are possible, in which case the main theorem becomes the following result.

MAIN THEOREM IN THE CASE $d=2$. Let $N(u)=\left(n_{1}(u)\right.$, let $\left.n_{2}(u)\right)=$ $\left(n_{1}, n_{2}\right)$, and assume that $N(u) / u \rightarrow\left(\alpha_{1}, \alpha_{2}\right)$. With the above notation, we have, for any integer $K>3$, the asymptotic formula

$$
\log \operatorname{det}^{*} \boldsymbol{\Delta}_{D T, N(u)}=n_{1} n_{2} \frac{4 G}{\pi}+\log \left(n_{1} n_{2}\right)+\log \left(\left|\eta\left(i \alpha_{2} / \alpha_{1}\right)\right|^{4} \alpha_{2} / \alpha_{1}\right)
$$

$$
+\sum_{k=1}^{K-3} F_{k}(u)+O\left(u^{-K}\right)
$$

as $u \rightarrow \infty$, where $G$ is the classical Catalan constant, $\eta$ denotes Dedekind's eta function, and the functions $F_{k}(u)$ are explicitly computable and satisfy the asymptotic bound $F_{k}(u)=O\left(u^{-k-2}\right)$ as $u \rightarrow \infty$.

The main theorem (5) comes from studying the special value $s=0$ in (4). The analysis we develop in the proof of (5) extends to prove the asymptotic behavior of (4) for all $s \in \mathbf{C}$ with $\operatorname{Re}\left(s^{2}\right)>0$, as well as for special values of the spectral zeta function $\sum_{j} \Lambda_{j}^{-w}$ for $w \in \mathbf{C}$ with $\operatorname{Re}(w)>0$. In Section 7.3, we prove the following theorem.

THEOREM. With notation as above, let $\zeta_{A}$ be the spectral zeta function on the real torus $A \mathbf{Z}^{d} \backslash \mathbf{R}^{d}$. Then for any $w \in \mathbf{C}$ with $\operatorname{Re}(w)>0$, we have that

$$
\begin{aligned}
& \lim _{u \rightarrow \infty} u^{-2 w}\left(\zeta_{N}(w)-\frac{V(N)}{\Gamma(w)} \int_{0}^{u^{2}}\left(e^{-2 t} I_{0}(2 t)\right)^{d} t^{w} \frac{d t}{t}\right) \\
& \quad=\zeta_{A}(w)-\frac{V(A)}{(4 \pi)^{d / 2}(w-d / 2) \Gamma(w)} .
\end{aligned}
$$

In particular, for any $w \in \mathbf{C}$ with $\operatorname{Re}(w)>d / 2$, we have

$$
\lim _{u \rightarrow \infty}\left(u^{-2 w} \zeta_{N(u)}(w)\right)=\zeta_{A}(w)
$$




\subsection{Comparisons with known results}

The case $d=2$ has been studied since at least the 1960s by physicists, starting with Kasteleyn's celebrated computation [16] of the lead term $4 G / \pi$. The next-order terms in the asymptotic expansion were later obtained by Barber (see [9, references therein]; see also [9, (3.18), for the precise statements]). Going further, [9] establishes the error term $O\left(u^{-1}\right)$, while our explicit error term expansion begins with a term of order $O\left(u^{-3}\right)$. The authors in [9] prove their results by studying the asymptotic behavior of the spectrum of the Laplacian, as opposed to the asymptotic behavior of the heat kernel and its integral transforms, which is the approach we take in the present article. Subsequent authors have studied asymptotics of the Laplacian on more general subgraphs of $\mathbf{Z}^{2}$ (see, e.g., [2], [17]). For general $d \geq 2$, the articles [2], [4], [11], [24], and [25] have discussions which give the lead-term asymptotics in (5). However, there is no discussion in these articles or elsewhere regarding the next-order term in the asymptotic expansion in $(5)$.

As stated, in Sections 7.3 and 7.4 we apply our analysis to study the asymptotic behavior of the families of spectral zeta functions and EpsteinHurwitz zeta functions through degeneration. These problems are considered in [9] in the case $d=2$ for general arguments for the Epstein-Hurwitz zeta function and for the special values $w=1$ and $w=2$ of the spectral zeta function (see Section 7.4 for a clarification of the notation). Specifically, [9, (3.24)] asserts that, "after a rather long algebra," one has the asymptotic formula

$$
\begin{aligned}
\sum_{k \neq 0} \frac{1}{\Lambda_{k}^{2} \sim} & \left(\frac{\alpha_{1} u \alpha_{2} u}{2 \pi}\right)^{2}\left(\frac{1}{2^{4} \cdot 45}\left(2 \pi \alpha_{1} / \alpha_{2}\right)^{2}+\frac{\zeta(3)}{2\left(2 \pi \alpha_{1} / \alpha_{2}\right)}\right. \\
& \left.+\frac{1}{2 \pi \alpha_{1} / \alpha_{2}} \sum_{n \geq 1} \frac{1}{n^{3}} \frac{e^{-2 \pi \alpha_{1} / \alpha_{2} n}}{1-e^{-2 \pi \alpha_{1} / \alpha_{2} n}}+\sum_{n \geq 1} \frac{1}{n^{2}} \frac{e^{-2 \pi \alpha_{1} / \alpha_{2} n}}{\left(1-e^{-2 \pi \alpha_{1} / \alpha_{2} n}\right)^{2}}\right)
\end{aligned}
$$

as $u \rightarrow \infty$. For comparison, our result (8) specialized to $d=w=2$ yields the asymptotic formula

$$
\sum_{k \neq 0} \frac{1}{\Lambda_{k}^{2}} \sim \frac{u^{4}}{(2 \pi)^{4}} \sum_{(n, m) \neq(0,0)} \frac{1}{\left(\left(n / \alpha_{1}\right)^{2}+\left(m / \alpha_{2}\right)^{2}\right)^{2}}
$$

as $u \rightarrow \infty$. Going further, the current literature contains only a handful of other considerations beyond (9), all in the case of $d=2$, whereas our result is completely general. 
As an aside, note that by equating the lead terms in (9) and (10) and setting $y=\alpha_{1} / \alpha_{2}$, we arrive at the formula

$$
\begin{aligned}
& \sum_{(n, m) \neq(0,0)} \frac{1}{\left(n^{2}+(m y)^{2}\right)^{2}} \\
& =\left(\frac{2 \pi}{y}\right)^{2}\left(\frac{(2 \pi y)^{2}}{2^{4} \cdot 45}+\frac{\zeta(3)}{4 \pi y}\right. \\
& \left.\quad+\frac{1}{2 \pi y} \sum_{n \geq 1} \frac{1}{n^{3}} \frac{e^{-2 \pi y n}}{1-e^{-2 \pi y n}}+\sum_{n \geq 1} \frac{1}{n^{2}} \frac{e^{-2 \pi y n}}{\left(1-e^{-2 \pi y n}\right)^{2}}\right) .
\end{aligned}
$$

A direct proof of (11) can be obtained by taking the Fourier expansion of the nonholomorphic Eisenstein series $E(z, s)$ for $\operatorname{SL}(2, \mathbf{Z})$ with $z=i y$ and $s=2$, using the evaluation of the $K$-Bessel function $K_{s-1 / 2}$ in terms of exponential functions when $s=2$. We thank Cormac O'Sullivan for clarifying this point for us, and we refer the interested reader to his forthcoming article ([20]) for a systematic presentation of identities of this form.

In Riemannian geometry, the determinant of the Laplacian obtained through zeta-function regularization has been used extensively as a height function on moduli space (see, e.g., [5], [19]). We view the asymptotic expansion in (5) as establishing a precise connection to the well-established notion of complexity from graph theory (compare with the discussion in [22, page 619$]$ or [17, page 242]). Another context where the number of spanning trees in discrete tori appears is in the study of sandpile models [3]. Going beyond the setting of discrete and real tori, we are optimistic that the point of view taken in the present article will extend to address problems in the study of other families of Cayley graphs of discrete, possibly infinite, groups.

\subsection{Outline of the article}

In Section 2, we establish notation and present background material from elsewhere in the mathematics literature.

In Section 3, we study the theta function (3) associated to the action of the discrete Laplacian on functions on $D T_{N}=\prod_{j=1}^{d} n_{j} \mathbf{Z} \backslash \mathbf{Z}$. Following the results from [15], we begin with the inversion formula (3) obtained by expressing the heat kernel on $\mathbf{Z}^{d}$ in terms of $I$-Bessel functions and then periodize to obtain an expression for the heat kernel on the discrete torus. The main result in Section 3 is Theorem 3.6, stated in (4) above, which computes the Gauss transform (Laplace transform with a quadratic change of variables) of the heat kernel on $D T_{N}$. By taking a special value of the Gauss 
transform, we obtain an expression for the determinant of the Laplacian on $D T_{N}$ in terms of integral transforms of $I$-Bessel functions.

In Section 4, we prove general bounds for $I$-Bessel functions, building from the fundamental estimates proved in [21]. Many of the computations in Section 4 involve rescaled $I$-Bessel functions. For instance, we establish asymptotic behavior (Proposition 4.7) and uniform bounds (Lemma 4.6) in the parameter $u$ of

$$
f(u ; x, t)=u e^{-u^{2} t} I_{u x}\left(u^{2} t\right) .
$$

In particular, we prove that as $u$ tends to infinity, $f(u ; x, t)$ approaches the heat kernel on $\mathbf{R}$ associated to a certain scaling of the usual Laplacian.

In Section 5 we define and study spectral asymptotics on degenerating sequences of discrete tori. Theorem 5.8, and its reformulation in Remark 5.9 , proves the main result of this article as stated in (5), which is the asymptotic behavior of the determinant of the Laplacian on a degenerating family of discrete tori. The two key steps are to first employ the change of variable $t \rightarrow u^{2} t$ and then utilize a careful decomposition of the Bessel integrals involved. The various integrals are then analyzed individually and matched up with terms in the meromorphic continuation of the spectral determinant on the continuous side.

Whereas we highlight Theorem 5.8 as the main result of the present article, there are many other applications of the analysis from Sections 3 and 4 that go beyond the study of the determinant of the Laplacian, which is the focus of attention in Section 5 .

In Section 6, we deduce our main theorem for $d=2$ and show that it agrees with the work in [9]. Moreover, we show how to obtain an explicit expansion of the error term. This does not appear in previous work and could be of importance when comparing heights of discrete tori and corresponding continuous tori.

The main points we address in Section 7 are examination of the numerical evaluation for the lead term in various asymptotic expansions for arbitrary dimension, determination of asymptotic behavior of spectral zeta functions and Epstein-Hurwitz zeta functions for general dimension, and investigation of the notational changes needed to consider general sequences of degenerating discrete tori. 


\section{$\S 2$. Preliminary material}

The purpose of this section is to establish notation and recall relevant material from elsewhere in the mathematics literature.

\subsection{Basic notation}

We use $t \in \mathbf{R}^{+}$to denote a positive real variable, and we use $x \in \mathbf{Z}$ to denote an integer variable. For any integer $n \geq 1$, we call the quotient space $n \mathbf{Z} \backslash \mathbf{Z}$ a discrete circle, and a finite product of discrete circles a discrete torus. The product of $d$ discrete circles formed with the integers $n_{1}, \ldots, n_{d}$ will be denoted by $D T_{N}$, where $N=\left(n_{j}\right)_{j=1, \ldots, d}$.

For any function $f: \mathbf{Z} \rightarrow \mathbf{R}$, we define the Laplacian $\Delta_{\mathbf{Z}}$ by

$$
\Delta_{\mathbf{Z}} f(x)=2 f(x)-(f(x+1)+f(x-1)) .
$$

The Laplacian on $\mathbf{Z}^{d}$ is the sum of $d$ Laplacians $\Delta_{\mathbf{Z}}$; one for each coordinate. The spectrum of the Laplacian $\Delta_{\mathbf{Z}}$ acting on function on $n \mathbf{Z} \backslash \mathbf{Z}$ is easily computable (see, e.g., [15]); hence the spectrum on $D T_{N}$ is simply the set of sums of eigenvalues for each discrete circle. Specifically, let $\left\{\Lambda_{j}\right\}$ denote the set of eigenvalues of $\Delta$ acting on function on $D T_{N}$. Then, with our normalization of the Laplacian,

$$
\left\{\Lambda_{j}\right\}=\left\{2 d-2 \cos \left(2 \pi k_{1} / n_{1}\right)-\cdots-2 \cos \left(2 \pi k_{d} / n_{d}\right)\right\},
$$

with $k_{1}=0, \ldots, n_{1}-1, \ldots, k_{d}=0, \ldots, n_{d}-1$. Let $V(N)=n_{1}, \ldots, n_{d}$, which can be viewed as a volume of $D T_{N, d}$. There are $V(N)$ eigenvalues of the Laplacian on $D T_{N}$, and $V(N)-1$ of the eigenvalues are nonzero.

The discrete torus $D T_{N}$ gives rise to a graph by inserting an edge between two points which have a single component that differs by one (i.e., nearest neighbor). This is the Cayley graph of the group $\prod_{j=1}^{d} n_{j} \mathbf{Z} \backslash \mathbf{Z}$ with respect to the generators corresponding to the standard basis vectors of $\mathbf{Z}^{d}$. A spanning tree is a subgraph such that given any two vertices in $D T_{N}$, there is precisely one path within the subgraph that connects the two vertices. The matrixtree theorem (see, e.g., [12]) asserts that

$$
\text { \# of spanning trees }=\frac{1}{V(N)} \prod_{\Lambda_{j} \neq 0} \Lambda_{j} .
$$

We will use the notation

$$
\operatorname{det}^{*} \boldsymbol{\Delta}_{D T, N}=\prod_{\Lambda_{j} \neq 0} \Lambda_{j}
$$


where $\operatorname{det}^{*} \boldsymbol{\Delta}_{D T, N}$ denotes the determinant of the Laplacian on the discrete torus $D T_{N}$ omitting the zero eigenvalue.

\subsection{The $I$-Bessel function}

Classically, the $I$-Bessel function $I_{x}(t)$ is defined as a solution to the differential equation

$$
t^{2} \frac{d^{2} w}{d t^{2}}+t \frac{d w}{d t}-\left(t^{2}+x^{2}\right)=0 .
$$

For integer values of $x$, it is immediately shown that $I_{x}=I_{-x}$, and for positive integer values of $x$, we have the series representation

$$
I_{x}(t)=\sum_{n=0}^{\infty} \frac{(t / 2)^{2 n+x}}{n ! \Gamma(n+1+x)},
$$

as well as the integral representation

$$
I_{x}(t)=\frac{1}{\pi} \int_{0}^{\pi} e^{t \cos (\theta)} \cos (\theta x) d \theta .
$$

The mathematics literature contains a vast number of articles and monographs which study the many fascinating properties and manifestations of the $I$-Bessel functions, as well as other Bessel functions. As demonstrated in the analysis in [15], basic to our considerations is the relation

$$
I_{x+1}(t)+I_{x-1}(t)=2 \frac{d}{d t} I_{x}(t)
$$

which easily can be derived from the integral representation and simple trigonometric identities.

\subsection{Universal bounds for the $I$-Bessel function}

The elementary definition of the $I$-Bessel function leads to a number of precise expressions for $I_{x}(t)$, two of which are stated in Section 2.2. Unfortunately, the explicit identities do not easily lead to viable estimates for $I_{x}(t)$. Beginning with the differential equation which characterizes $I_{x}(t)$, Pal'tsev [21] was able to derive very precise upper and lower bounds for the $I$-Bessel function, which we now state. Let

$$
g_{x}(t)=\sqrt{\left(x^{2}+t^{2}\right)}+x \log \left(\frac{t}{x+\sqrt{\left(x^{2}+t^{2}\right)}}\right) .
$$


Then for all $t>0$ and $x \geq 2$ we have that

$$
e^{-1 /\left(2 \sqrt{\left(x^{2}+t^{2}\right)}\right)} \leq \sqrt{(2 \pi)} \cdot\left(x^{2}+t^{2}\right)^{1 / 4} I_{x}(t) e^{-g_{x}(t)} \leq e^{1 /\left(2 \sqrt{\left(x^{2}+t^{2}\right)}\right)} .
$$

These bounds play an important role in this article. Indeed, in Section 4, we study the function $g_{x}(t)-t$ in order to obtain elementary bounds for $\sqrt{t} e^{-t} I_{x}(t)$, which will be vital when establishing our main result.

\subsection{Heat kernels and zeta functions}

In [15], the authors established a theta inversion formula obtained from studying the heat kernel associated to the Laplacian on $n \mathbf{Z} \backslash \mathbf{Z}$. Let $K_{\mathbf{Z}}(t, x)$ be a function on $\mathbf{Z}$ such that

$$
\Delta_{\mathbf{Z}} K_{\mathbf{Z}}(t, x)+\frac{\partial}{\partial t} K_{\mathbf{Z}}(t, x)=0
$$

and

$$
\lim _{t \rightarrow 0} K_{\mathbf{Z}}(t, x)= \begin{cases}1 & \text { if } x=0 \\ 0 & \text { if } x \neq 0\end{cases}
$$

General results from [7] and [6] prove the existence and uniqueness of $K_{\mathbf{Z}}(t, x)$, and as shown, for example, in [15], we have that

$$
K_{\mathbf{Z}}(t, x)=e^{-2 t} I_{x}(2 t)
$$

The existence and uniqueness theorems for heat kernels on graphs apply to heat kernels on $n \mathbf{Z} \backslash \mathbf{Z}$. Bounds for the $I$-Bessel function from Section 2.3 imply that the series

$$
\sum_{k=-\infty}^{\infty} e^{-2 t} I_{k n+x}(2 t)
$$

converges and hence is equal to the heat kernel on $n \mathbf{Z} \backslash \mathbf{Z}$, which we denote by $K_{n \mathbf{Z} \backslash \mathbf{Z}}(t, x)$. Denote the eigenvalues on $n \mathbf{Z} \backslash \mathbf{Z}$ and corresponding eigenfunctions by $\left\{\lambda_{n, j}\right\}$ and $\left\{\phi_{n, j}\right\}$, respectively. This leads to the identity

$$
\sum_{j=0}^{n-1} e^{-t \lambda_{n, j}} \phi_{n, j}(x) \phi_{n, j}(0)=\sum_{k=-\infty}^{\infty} e^{-2 t} I_{k n+x}(2 t) .
$$

In the case $n=1$, this yields a classical identity which expresses an infinite sum of $I$-Bessel functions as an exponential function. The heat kernel on 
$D T_{N}$ and corresponding theta inversion formula are obtained by taking the $d$-fold product of (17).

\subsection{Spectral analysis on real tori}

Given a positive definite $d \times d$ matrix $A$, let $\mathrm{RT}_{A}$ denote the real torus $A \mathbf{Z}^{d} \backslash \mathbf{R}^{d}$. On $\mathbf{R}$ we use the variable $y$ to denote the standard global coordinate, from which we have the Laplacian

$$
\Delta_{\mathbf{R}} f(y)=-\frac{d^{2}}{d y^{2}} f(y)
$$

and corresponding heat kernel $K_{\mathbf{R}}(t, y)$ which is uniquely characterized by the conditions

$$
\Delta_{\mathbf{R}} K_{\mathbf{R}}(t, y)+\frac{\partial}{\partial t} K_{\mathbf{R}}(t, y)=0
$$

and

$$
\int_{\mathbf{R}} K_{\mathbf{R}}(t, x-y) f(y) d y \rightarrow f(x), \quad \text { as } t \rightarrow 0,
$$

for any smooth, real-valued function $f$ on $\mathbf{R}$ with compact support. The set of eigenvalues of the Laplacian on $\mathrm{RT}_{A}$ is given by $\left\{(2 \pi)^{2 t} m A^{*} m\right\}$ for $m \in$ $\mathbf{Z}^{d}$, where $A^{*}$ is the dual lattice to $A$. The eigenfunctions of the Laplacian are expressible as exponential functions. The theta function

$$
\Theta_{A}(t)=\sum_{m \in \mathbf{Z}^{d}} e^{-(2 \pi)^{2} \cdot t} m A^{*} m \cdot t
$$

with $t>0$, is the trace of the heat kernel on $\mathrm{RT}_{A}$. The asymptotic behavior of the theta function is well known, namely, that

$$
\Theta_{A}(t)=V(A)(4 \pi t)^{-d / 2}+O\left(e^{-c / t}\right), \quad \text { for some } c>0 \text { as } t \rightarrow 0
$$

and

$$
\Theta_{A}(t)=1+O\left(e^{-c t}\right), \quad \text { for some } c>0 \text { as } t \rightarrow \infty .
$$

\subsection{Regularized determinants on real tori}

In very general circumstances, the spectral zeta function is defined as the Mellin transform $\mathbf{M}$ of the theta function formed with the nonzero eigenvalues. Specifically, for $s \in \mathbf{C}$ with $\operatorname{Re}(s)>d / 2$, we define the spectral zeta function

$$
\zeta_{A}(s)=\mathbf{M} \Theta_{A}(s)=\frac{1}{\Gamma(s)} \int_{0}^{\infty}\left(\Theta_{A}(t)-1\right) t^{s} \frac{d t}{t} .
$$


The integral in (18) converges for $\operatorname{Re}(s)>d / 2$. Let us write

$$
\begin{aligned}
\zeta_{A}(s)= & \frac{1}{\Gamma(s)} \int_{0}^{1}\left(\Theta_{A}(t)-V(A)(4 \pi t)^{-d / 2}\right) t^{s} \frac{d t}{t} \\
& +\frac{1}{\Gamma(s)} \int_{0}^{1}\left(V(A)(4 \pi t)^{-d / 2}-1\right) t^{s} \frac{d t}{t} \\
& +\frac{1}{\Gamma(s)} \int_{1}^{\infty}\left(\Theta_{A}(t)-1\right) t^{s} \frac{d t}{t}
\end{aligned}
$$

Going further, we can carry out the integral in the second term to get

$$
\begin{aligned}
\zeta_{A}(s)= & \frac{1}{\Gamma(s)} \int_{0}^{1}\left(\Theta_{A}(t)-V(A)(4 \pi t)^{-d / 2}\right) t^{s} \frac{d t}{t} \\
& +(4 \pi)^{-d / 2} \frac{V(A)}{(s-d / 2) \Gamma(s)}-\frac{1}{\Gamma(s+1)} \\
& +\frac{1}{\Gamma(s)} \int_{1}^{\infty}\left(\Theta_{A}(t)-1\right) t^{s} \frac{d t}{t} .
\end{aligned}
$$

By virtue of the asymptotic behavior of $\Theta_{A}(t)$ as $t \rightarrow 0$ and $t \rightarrow \infty$, expression (20) provides a meromorphic continuation of $\zeta_{A}(s)$ to $s \in \mathbf{C}$. Therefore, using (20), we can study the behavior of $\zeta_{A}(s)$ near $s=0$. The integrals are holomorphic near $s=0$, and $1 / \Gamma(s)=s+O\left(s^{2}\right)$ near $s=0$, so then we have that $\zeta_{A}(0)=-1$, which is a point that will be used later. In particular, we have that

$$
\begin{aligned}
\zeta_{A}^{\prime}(0)= & \int_{0}^{1}\left(\Theta_{A}(t)-V(A)(4 \pi t)^{-d / 2}\right) \frac{d t}{t}+\Gamma^{\prime}(1) \\
& -\frac{2}{d} V(A)(4 \pi)^{-d / 2}+\int_{1}^{\infty}\left(\Theta_{A}(t)-1\right) \frac{d t}{t},
\end{aligned}
$$

which will play an important role in the proof of our main result.

\subsection{Kronecker's limit formula}

The special value $\zeta_{A}^{\prime}(0)$ has a unique place in classical analytic number theory, and the evaluation of $\zeta_{A}^{\prime}(0)$ in terms of modular forms is generally referred to as Kronecker's limit formula, which we now describe. The content of this section has its origins in [10], and the discussion we present here comes directly from [8]. 
Let $Q$ denote a $d \times d$ positive definite matrix, and let $u \in \mathbf{R}^{d}$ any vector. If we write $Q=\left(q_{i, j}\right)$, then the quadratic form associated to $Q$ is defined by

$$
Q(u)=\sum_{i, j} q_{i, j} u_{i} u_{j}
$$

The Epstein zeta function associated to $Q$ is defined for $\operatorname{Re}(s)>d / 2$ by the convergent series

$$
Z(s, Q)=\sum_{m \in \mathbf{Z}^{d} \backslash\{0\}} Q(m)^{-s} .
$$

From the discussion in Sections 2.4 and 2.5, the Epstein zeta function is, up to a multiplicative factor of $(2 \pi)^{-2 s}$, the spectral zeta function associated to the real torus $\mathrm{RT}_{A}$ with $A=Q^{*}$, where $Q^{*}$ is the dual lattice to $Q$. It can be shown that $Z(s, Q)$ admits a meromorphic continuation and the functional equation

$$
\pi^{-s} \Gamma(s) Z\left(s, Q^{-1}\right)=(\operatorname{det} Q)^{1 / 2} \pi^{s-d / 2} \Gamma(d / 2-s) Z(d / 2-s, Q) .
$$

The Iwasawa decomposition of $Q$ asserts that $Q$ can be uniquely expressed as

$$
Q=\left(\begin{array}{cc}
1 & 0 \\
-{ }^{t} x & I_{d-1}
\end{array}\right)\left(\begin{array}{cc}
y^{-1} & 0 \\
0 & Y
\end{array}\right)\left(\begin{array}{cc}
1 & -x \\
0 & I_{d-1}
\end{array}\right)
$$

with $y \in \mathbf{R}_{+}, x \in \mathbf{R}^{d-1}$, and $Y$ a $(d-1) \times(d-1)$ positive definite matrix; here, $I_{d-1}$ denotes the $(d-1) \times(d-1)$ identity matrix, and we think of $x \in \mathbf{R}^{d-1}$ and $y \in \mathbf{R}$ as row vectors. For $m \in \mathbf{R}^{d-1}$, one defines

$$
Q\{m\}=m \cdot x+i \sqrt{y Y(m)},
$$

which is a complex number with $\operatorname{Im}(Q\{m\})>0$ unless $m=0$. With all this, the generalization of the classical Kronecker limit formula for $Z(s, Q)$ is the identity

$$
Z^{\prime}(0, Q)=-2 \pi \sqrt{y} Z(1 / 2, Y)-\log \left|(2 \pi)^{2} y \prod_{m \in \mathbf{Z}^{n-1} \backslash\{0\}}\left(1-e^{2 \pi i Q\{m\}}\right)^{4}\right| .
$$

In [8] the authors study $\exp \left(-Z^{\prime}(0, Q)\right)$ as a generalization of the classical Dedekind eta function and prove an analogue of the Chowla-Selberg formula which relates $\exp \left(-Z^{\prime}(0, Q)\right)$ to special values of higher order gamma func- 
tions and special values of certain $L$ functions. We refer the interested reader to [8] for additional results regarding the fascinating number theoretic and automorphic aspects of (22).

\subsection{Mellin transform and inversion}

Let $f_{x}(t)=e^{-t / 2} I_{x}(t / 2)$. Known transform identities include the formula

$$
\tilde{f}_{x}(s)=\int_{0}^{\infty} e^{-t / 2} I_{x}(t / 2) t^{s} \frac{d t}{t}=\frac{\Gamma(s+x) \Gamma(1 / 2-s)}{\sqrt{\pi} \Gamma(x+1-s)} .
$$

The identity $(23)$ is valid for $-x<\operatorname{Re}(s)<1 / 2$. The inverse Mellin formula gives

$$
f_{x}(t)=\frac{1}{2 \pi i} \int_{(\sigma)} \tilde{f}_{x}(s) t^{-s} d s, \quad \text { for }-x<\sigma<1 / 2 .
$$

The Mellin transform of a product is computed via the convolution of two transforms, yielding the computations

$$
\begin{aligned}
\widetilde{f_{x} f_{y}}(s) & =\int_{0}^{\infty} f_{x}(t) f_{y}(t) t^{s} \frac{d t}{t} \\
& =\int_{0}^{\infty} f_{x}(t)\left[\frac{1}{2 \pi i} \int_{(\sigma)} \tilde{f}_{y}(z) t^{-z} d z\right] t^{s} \frac{d t}{t}, \quad \text { for }-y<\sigma<1 / 2 \\
& =\frac{1}{2 \pi i} \int_{(\sigma)} \tilde{f}_{y}(z)\left[\int_{0}^{\infty} f_{x}(t) t^{s-z} \frac{d t}{t}\right] d z, \quad \text { for }-x<\operatorname{Re}(s)-\sigma<1 / 2 \\
& =\frac{1}{2 \pi i} \int_{(\sigma)} \tilde{f}_{x}(s-z) \tilde{f}_{y}(z) d z .
\end{aligned}
$$

Therefore, we conclude that

$$
\begin{aligned}
\widetilde{f_{x} f_{y}}(s) & =\int_{0}^{\infty} e^{-t} I_{x}(t / 2) I_{y}(t / 2) t^{s} \frac{d t}{t} \\
& =\frac{1}{2 \pi i} \int_{(\sigma)} \frac{\Gamma(s-z+x) \Gamma(1 / 2+z-s) \Gamma(z+y) \Gamma(1 / 2-z)}{\sqrt{\pi} \Gamma(x+1+z-s) \sqrt{\pi} \Gamma(y+1-z)} d z \\
& =\frac{1}{2 \pi i} \int_{(\sigma)} \frac{\Gamma(s-z+x) \Gamma(1 / 2+z-s) \Gamma(z+y) \Gamma(1 / 2-z)}{\pi \Gamma(x+1+z-s) \Gamma(y+1-z)} d z .
\end{aligned}
$$

Using the bounds $I_{x}(t)=O\left(t^{x}\right)$ as $t \rightarrow 0$ and $e^{-t} I_{x}(t)=O\left(t^{-1 / 2}\right)$ as $t \rightarrow \infty$, the Mellin transform $\widetilde{f_{x} f_{y}}(s)$ is defined for $-x-y<\operatorname{Re}(s)<1$, so $(25)$ holds 
for $0<\sigma<\operatorname{Re}(s)<1 / 2$. If $x \neq 0$ or $y \neq 0$, we can take $s=0$ and write

$$
\begin{aligned}
& \int_{0}^{\infty} e^{-t}\left[I_{x}(t / 2) I_{y}(t / 2)\right] \frac{d t}{t} \\
& \quad=\frac{1}{2 \pi i} \int_{(1 / 4)} \frac{\Gamma(x-z) \Gamma(1 / 2+z) \Gamma(z+y) \Gamma(1 / 2-z)}{\pi \Gamma(x+1+z) \Gamma(y+1-z)} d z .
\end{aligned}
$$

In Section 6.2 below, we will extend (26) to the case $x=y=0$.

\subsection{Miscellaneous results}

For any $\varepsilon>0$ and $z \in \mathbf{C}$, Stirling's formula for the classical gamma function is the asymptotic relation

$$
\log \Gamma(z)=(z-1 / 2) \log z-z+(1 / 2) \log (2 \pi)+O(1 / z)
$$

as $z \rightarrow \infty$ provided $|\arg (z)|<\pi-\varepsilon$. In particular, one has for fixed $a$ and $b$ the asymptotic relation

$$
\frac{\Gamma(z+a)}{\Gamma(z+b)}=z^{a-b}+O\left(z^{a-b-1}\right), \quad \text { as } z \rightarrow \infty \text { provided }|\arg (z)|<\pi-\varepsilon .
$$

Further terms in the Stirling's formula can be computing (see, e.g., [13]), which then would imply further terms in the asymptotic expansion (27).

Throughout our work, we will use the elementary identity

$$
\log (w)=\int_{0}^{\infty}\left(e^{-t}-e^{-w t}\right) \frac{d t}{t}, \quad \text { for all } w \in \mathbf{C} \text { with } \operatorname{Re}(w)>0 .
$$

To prove this relation, one simply observes that both sides of the proposed identity vanish when $w=1$ and have first derivative equal to $1 / w$.

\section{$\S 3$. Zeta functions and determinants for discrete tori}

In this section we study the Gauss transform of the trace of the heat kernel associated to a discrete torus. We begin with Lemma 3.1, which recalls the theta inversion formula associated to a general discrete torus. From the theta inversion formula, we define the Gauss transform (Lemma 3.2). Using the group periodization representation of the heat kernel, we decompose the theta inversion formula into two summands: the identity term and the set of nonidentity terms. We study the Gauss transform of these two summands separately, ultimately arriving at an identity (Theorem 3.6) which expresses the determinant of the Laplacian on the discrete torus in terms of $I$-Bessel functions. 
LEMMA 3.1. Let $\theta_{N}(t)$ be the theta function associated to the d-dimensional discrete torus $N \mathbf{Z}^{d} \backslash \mathbf{Z}^{d}$, defined by

$$
\theta_{N}(t)=\sum_{\Lambda_{j}} e^{-\Lambda_{j} t}
$$

where

$$
\begin{gathered}
\left\{\Lambda_{j}\right\}=\left\{2 d-2 \cos \left(2 \pi m_{1} / n_{1}\right)-\cdots-2 \cos \left(2 \pi m_{d} / n_{d}\right):\right. \\
\left.0 \leq m_{i}<n_{i}, \text { for each } i=1,2, \ldots, d\right\}
\end{gathered}
$$

Then for all $t>0$, we have the identity

$$
\theta_{N}(t)=V(N) \sum_{K \in \mathbf{Z}^{d}} \prod_{1 \leq j \leq d} e^{-2 t} I_{n_{j} \cdot k_{j}}(2 t)
$$

where $K$ runs over $d$-tuples of integers $\left(k_{1}, \ldots, k_{d}\right)$.

Proof. We refer to [15] for the case $d=1$. From there, one gets the general case using that the heat kernel on a product space is equal to the product of the heat kernels, since the Laplacian on the product space is defined to be the sum of the Laplacians from each factor space.

Lemma 3.2. For all $s \in \mathbf{C}$ with $\operatorname{Re}\left(s^{2}\right)>0$, we have

$$
\begin{aligned}
\sum_{\Lambda_{j} \neq 0} \frac{2 s}{s^{2}+\Lambda_{j}}= & V(N) 2 s \int_{0}^{\infty} e^{-s^{2} t} e^{-2 d t} I_{0}(2 t)^{d} d t \\
& +2 s \int_{0}^{\infty} e^{-s^{2} t}\left[\theta_{N}(t)-V(N) e^{-2 d t} I_{0}(2 t)^{d}-1\right] d t
\end{aligned}
$$

Proof. By the definition of $\theta_{N}$, we can write

$$
\sum_{\Lambda_{j} \neq 0} e^{-\Lambda_{j} t}=V(N)\left(e^{-2 t} I_{0}(2 t)\right)^{d}+\left[\theta_{N}(t)-V(N) e^{-2 d t} I_{0}(2 t)^{d}-1\right] .
$$

Now simply multiply both sides of this identity by $2 s e^{-s^{2} t}$ and integrate with respect to $t$ on $(0, \infty)$. Asymptotic behavior of the integrands as $t \rightarrow 0$ and $t \rightarrow \infty$ easily implies that the resulting integrals are convergent for $s \in \mathbf{C}$ provided $\operatorname{Re}\left(s^{2}\right)>0$. 
LEMMA 3.3. The function

$$
f(s)=\sum_{\Lambda_{j} \neq 0} \log \left(s^{2}+\Lambda_{j}\right)
$$

is uniquely characterized by the differential equation

$$
\partial_{s} f(s)=\sum_{\Lambda_{j} \neq 0} \frac{2 s}{s^{2}+\Lambda_{j}}
$$

and the asymptotic relation

$$
f(s)=(V(N)-1) \cdot \log s^{2}+o(1), \quad \text { as } s \rightarrow \infty .
$$

Proof. The differential equation characterizes $f(s)$ up to an additive constant, which is uniquely determined by the stated asymptotic behavior.

Proposition 3.4. The function

$$
\mathcal{I}_{d}(s)=-\int_{0}^{\infty}\left(e^{-s^{2} t} e^{-2 d t} I_{0}(2 t)^{d}-e^{-t}\right) \frac{d t}{t}
$$

is uniquely characterized by the differential equation

$$
\partial_{s} \mathcal{I}_{d}(s)=2 s \int_{0}^{\infty} e^{-s^{2} t} e^{-2 d t} I_{0}(2 t)^{d} d t
$$

and the asymptotic relation

$$
\mathcal{I}_{d}(s)=\log s^{2}+o(1), \quad \text { as } s \rightarrow+\infty .
$$

Proof. For this, we write

$$
\mathcal{I}_{d}(s)=-\int_{0}^{\infty} e^{-s^{2} t} e^{-2 d t}\left(I_{0}(2 t)^{d}-1\right) \frac{d t}{t}-\int_{0}^{\infty}\left(e^{-s^{2} t} e^{-2 d t}-e^{-t}\right) \frac{d t}{t} .
$$

By (28),

$$
\int_{0}^{\infty}\left(e^{-s^{2} t} e^{-2 d t}-e^{-t}\right) \frac{d t}{t}=-\log \left(s^{2}+2 d\right)
$$

so then we have

$$
\mathcal{I}_{d}(s)=-\int_{0}^{\infty} e^{-s^{2} t} e^{-2 d t}\left(I_{0}(2 t)^{d}-1\right) \frac{d t}{t}+\log \left(s^{2}+2 d\right) .
$$

From this last expression, the asymptotic behavior as $s \rightarrow \infty$ is immediate. 
Proposition 3.5. The function

$$
\mathcal{H}_{N}(s)=-\int_{0}^{\infty}\left(e^{-s^{2} t}\left[\theta_{N}(t)-V(N) e^{-2 d t} I_{0}(2 t)^{d}-1\right]+e^{-t}\right) \frac{d t}{t}
$$

is uniquely characterized by the differential equation

$$
\partial_{s} \mathcal{H}_{N}(s)=2 s \int_{0}^{\infty} e^{-s^{2} t}\left[\theta_{N}(t)-V(N) e^{-2 d t} I_{0}(2 t)^{d}-1\right] d t
$$

and the asymptotic relation

$$
\mathcal{H}_{N}(s)=-\log s^{2}+o(1), \quad \text { as } s \rightarrow+\infty
$$

Proof. For this, we write

$$
\begin{aligned}
\mathcal{H}_{N}(s) & =-\int_{0}^{\infty} e^{-s^{2} t}\left(\theta_{N}(t)-V(N) e^{-2 d t} I_{0}(2 t)^{d}\right) \frac{d t}{t}+\int_{0}^{\infty}\left(e^{-s^{2} t}-e^{-t}\right) \frac{d t}{t} \\
& =-\int_{0}^{\infty} e^{-s^{2} t}\left(\theta_{N}(t)-V(N) e^{-2 d t} I_{0}(2 t)^{d}\right) \frac{d t}{t}-\log \left(s^{2}\right) .
\end{aligned}
$$

Clearly, the integral in (29) approaches zero as $s$ approaches infinity, which completes the proof of the stated asymptotic relation.

Theorem 3.6. For any $s \in \mathbf{C}$ with $\operatorname{Re}\left(s^{2}\right)>0$, we have the relation

$$
\sum_{\Lambda_{j} \neq 0} \log \left(s^{2}+\Lambda_{j}\right)=V(N) \mathcal{I}_{d}(s)+\mathcal{H}_{N}(s)
$$

Letting $s \rightarrow 0$, we have the identity

$$
\log \left(\prod_{\Lambda_{j} \neq 0} \Lambda_{j}\right)=V(N) \mathcal{I}_{d}(0)+\mathcal{H}_{N}(0)
$$

where

$$
\mathcal{I}_{d}(0)=-\int_{0}^{\infty}\left(e^{-2 d t} I_{0}(2 t)^{d}-e^{-t}\right) \frac{d t}{t}
$$

and

$$
\mathcal{H}_{N}(0)=-\int_{0}^{\infty}\left(\theta_{N}(t)-V(N) e^{-2 d t} I_{0}(2 t)^{d}-1+e^{-t}\right) \frac{d t}{t}
$$


Proof. We begin with the relation from Lemma 3.2. By substituting from the differential equations from Lemma 3.3 and Propositions 3.4 and 3.5 and then integrating, we get

$$
\sum_{\Lambda_{j} \neq 0} \log \left(s^{2}+\Lambda_{j}\right)=V(N) \mathcal{I}_{d}(s)+\mathcal{H}_{N}(s)+C
$$

for some constant $C$. We now use the asymptotic behavior relations as $s \rightarrow$ $\infty$ from Lemma 3.3 and Propositions 3.4 and 3.5 to show that $C=0$. From the series expansion (13), we have

$$
e^{-2 d t} I_{0}(2 t)^{d}-e^{-t}=O(t), \quad \text { as } t \rightarrow 0
$$

Lemma 4.1 gives

$$
e^{-2 d t} I_{0}(2 t)^{d}=O\left(t^{-d}\right), \quad \text { as } t \rightarrow \infty
$$

so then the integrand in the definition of $\mathcal{I}_{d}(0)$ is $L^{1}(0, \infty)$ with respect to $d t / t$. Concerning the integrand in the definition of $\mathcal{H}_{N}(0)$, we have

$$
\theta_{N}(t)-V(N) e^{-2 d t} I_{0}(2 t)^{d}=O(t), \quad \text { as } t \rightarrow 0
$$

and

$$
e^{-t}-1=O(t), \quad \text { as } t \rightarrow 0,
$$

so then the integrand in the definition of $\mathcal{H}_{N}(0)$ is in $L^{1}(0,1)$ with respect to $d t / t$. Furthermore, we have

$$
\theta_{N}(t)-1=O\left(e^{-c t}\right), \quad \text { as } t \rightarrow \infty \text { for some } c>0
$$

and

$$
V(N) e^{-2 d t} I_{0}(2 t)^{d}-e^{-t}=O\left(t^{-d}\right), \quad \text { as } t \rightarrow \infty
$$

so then the integrand in the definition of $\mathcal{H}_{N}(0)$ is in $L^{1}(1, \infty)$ with respect to $d t / t$. With all this, we have that all functions in (30) are continuous and well defined for $s \in \mathbf{R}_{\geq 0}$, so we simply need to evaluate at $s=0$ to complete the proof. 


\section{$\S 4$. Bounds and asymptotic formulas for Bessel functions}

In this section we prove bounds for individual $I$-Bessel functions. For technical reasons, it is necessary to separately establish bounds for $I_{0}$ and for $I_{x}$ for $x>0$. As we show in Section 5 , it is necessary to consider the function

$$
u \cdot e^{-u^{2} t} I_{n(u)}\left(u^{2} t\right)
$$

for $u \geq 1$ and where $n(u) / u \rightarrow x$. The asymptotic behavior as $u \rightarrow \infty$ is given in Proposition 4.7; uniform bounds are established in Lemma 4.1 when $x=0$ and in Corollary 4.4 and Lemma 4.6 when $x>0$. Although we restrict our attention to integers $x \geq 0$, we recall that $I_{x}=I_{-x}$, so the results we prove apply for all $x \in \mathbf{N}$.

Lemma 4.1. For any $\varepsilon<\pi / 2$ and $t>0$, we have the bounds

$$
\begin{aligned}
0 \leq e^{-t} I_{0}(t) & \leq C \cdot t^{-1 / 2}, \\
\text { where } C & =\frac{1}{\sqrt{\left(2-\varepsilon^{2} / 6\right) \pi}}+\frac{\pi-\varepsilon}{\pi} \cdot \frac{1}{\sqrt{\left(1-\varepsilon^{2} / 12\right) \varepsilon^{2} e}} .
\end{aligned}
$$

Proof. The positivity of $I_{0}(t)$ follows immediately from the series expansion stated in Section 2.2. From the integral representation, choose any $\varepsilon \in(0, \pi / 2)$ and write

$$
e^{-t} I_{0}(t)=\frac{1}{\pi} \int_{0}^{\varepsilon} e^{-t(1-\cos (u))} d u+\frac{1}{\pi} \int_{\varepsilon}^{\pi} e^{-t(1-\cos (u))} d u .
$$

There exists $c(\varepsilon)>0$ such that $1-\cos (u) \geq c(\varepsilon) u^{2}$ for $u \in[0, \varepsilon]$. With this, for the first integral we have

$$
\frac{1}{\pi} \int_{0}^{\varepsilon} e^{-t(1-\cos (u))} d u \leq \frac{1}{\pi} \int_{0}^{\varepsilon} e^{-c(\varepsilon) t u^{2}} d u \leq \frac{1}{\sqrt{4 c(\varepsilon) \pi t}} .
$$

For the second integral, we trivially have

$$
\frac{1}{\pi} \int_{\varepsilon}^{\pi} e^{-t(1-\cos (u))} d u \leq \frac{\pi-\varepsilon}{\pi} e^{-t(1-\cos (\varepsilon))} \leq \frac{\pi-\varepsilon}{\pi} e^{-c(\varepsilon) t \varepsilon^{2}} .
$$

Combining, we have

$$
0 \leq e^{-t} I_{0}(t) \leq \frac{1}{\sqrt{4 c(\varepsilon) \pi t}}+\frac{\pi-\varepsilon}{\pi} e^{-c(\varepsilon) t \varepsilon^{2}} .
$$


Since $\varepsilon<\pi / 2$ and $u \leq \varepsilon$, we have the bound

$$
1-\cos (u) \geq u^{2} / 2-u^{4} / 24 \geq\left(1 / 2-\varepsilon^{2} / 24\right) u^{2},
$$

so we may take $c(\varepsilon)=\left(1 / 2-\varepsilon^{2} / 24\right)$. Therefore,

$$
0 \leq e^{-t} I_{0}(t) \leq \frac{1}{\sqrt{\left(2-\varepsilon^{2} / 6\right) \pi t}}+\frac{\pi-\varepsilon}{\pi} e^{-\left(1 / 2-\varepsilon^{2} / 24\right) \varepsilon^{2} \cdot t} .
$$

Using elementary calculus, one shows that

$$
t^{1 / 2} e^{-a t} \leq 1 / \sqrt{2 a e}
$$

so then

$$
\begin{aligned}
& 0 \leq e^{-t} I_{0}(t) \leq C \cdot t^{-1 / 2} \\
& \text { where } C=\frac{1}{\sqrt{\left(2-\varepsilon^{2} / 6\right) \pi}}+\frac{\pi-\varepsilon}{\pi} \cdot \frac{1}{\sqrt{\left(1-\varepsilon^{2} / 12\right) \varepsilon^{2} e}}
\end{aligned}
$$

REMARK 4.2. Directly from Lemma 4.1, we have that

$$
u e^{-u^{2} t} I_{0}\left(u^{2} t\right) \leq u \cdot C \cdot\left(u^{2} t\right)^{-1 / 2}=C \cdot t^{-1 / 2},
$$

so we indeed have established the uniform upper bound as claimed. Also, for our purposes, it is not necessary to optimize (31) through a judicious choice of $\varepsilon$. Numerically, one can show that by taking $\varepsilon=1.5$, which is allowed since we only required that $\varepsilon<\pi / 2$, we have that $C=0.676991 \ldots$ One point we use later (see Section 7.1) is that $C<1 / \sqrt{2}<1$. We use the numerical verification of this bound and omit the theoretical proof from our analysis, noting that the estimate for $C$ indeed can be proved from (31).

Lemma 4.3. For fixed $x \geq 1$ and $t>0$, consider the function

$$
h_{x}(t)=\sqrt{\left(x^{2}+t^{2}\right)}-t+x \log \left(\frac{t}{x+\sqrt{\left(x^{2}+t^{2}\right)}}\right) .
$$

Then we have the bound

$$
\exp \left(h_{x}(t)\right) \leq\left(\frac{t}{t+x}\right)^{x / 2}
$$

By continuity, the inequality also holds when $t=0$. 
Proof. To begin, observe that

$$
h_{x}(t)=\frac{x^{2}}{t+\sqrt{\left(x^{2}+t^{2}\right)}}+x \log \left(\frac{t}{x+\sqrt{\left(x^{2}+t^{2}\right)}}\right),
$$

which comes from the definition of $h_{x}(t)$ and by writing

$$
\sqrt{\left(x^{2}+t^{2}\right)}-t=\frac{x^{2}}{t+\sqrt{\left(x^{2}+t^{2}\right)}} .
$$

Therefore, we have

$$
\begin{aligned}
h_{x}(t) & =\frac{x^{2}}{t+\sqrt{\left(x^{2}+t^{2}\right)}}+x \log \left(\frac{t}{x+\sqrt{\left(x^{2}+t^{2}\right)}}\right) \\
& =\frac{x^{2}}{t+\sqrt{\left(x^{2}+t^{2}\right)}}+x \log \left(\frac{1}{t}\left(\sqrt{x^{2}+t^{2}}-x\right)\right) \\
& =\frac{x^{2}}{t+\sqrt{\left(x^{2}+t^{2}\right)}}+x \log \left(\sqrt{\left(1+\frac{x^{2}}{t^{2}}\right)}-\frac{x}{t}\right) \\
& =x\left(\frac{x / t}{1+\sqrt{\left((x / t)^{2}+1\right)}}+\log \left(\sqrt{\left(1+\frac{x^{2}}{t^{2}}\right)}-\frac{x}{t}\right)\right) .
\end{aligned}
$$

We now employ the change of variables

$$
u=\log \left(\sqrt{\left(1+\frac{x^{2}}{t^{2}}\right)}+\frac{x}{t}\right)
$$

which is equivalent to the relation $\sinh (u)=x / t$. Using the elementary identities

$$
1+(\sinh u)^{2}=(\cosh u)^{2}, \quad \sinh u=2 \sinh (u / 2) \cosh (u / 2)
$$

and

$$
1+\cosh u=2(\cosh (u / 2))^{2}, \quad \cosh u-\sinh u=e^{-u},
$$

we arrive at the expression

$$
h_{x}(t)=x(\tanh (u / 2)-u) .
$$

Trivially, since $u \geq 0$ we have that $\tanh (u / 2) \leq u / 2$, so then, for $x>0$,

$$
h_{x}(t) \leq-x \cdot u / 2=-(x / 2) \log \left(\sqrt{\left(1+\frac{x^{2}}{t^{2}}\right)}+\frac{x}{t}\right) \leq-(x / 2) \log \left(1+\frac{x}{t}\right) .
$$


With all this, we have that

$$
\exp \left(h_{x}(t)\right) \leq\left(1+\frac{x}{t}\right)^{-x / 2}=\left(\frac{t}{x+t}\right)^{x / 2},
$$

which completes the proof of the lemma.

Corollary 4.4. For any $t>0$ and integer $x \geq 0$, we have

$$
\sqrt{t} \cdot e^{-t} I_{x}(t) \leq\left(\frac{t}{t+x}\right)^{x / 2}=\left(1+\frac{x}{t}\right)^{-x / 2}
$$

Proof. We begin by considering $x \geq 2$, so then the analysis from Lemma 4.3 applies. Indeed, we use the trivial estimates

$$
\frac{1}{\sqrt{2 \pi}} e^{1 /\left(2 \sqrt{\left(x^{2}+t^{2}\right)}\right)} \leq \frac{e^{1 / 4}}{\sqrt{2 \pi}} \leq 1
$$

and

$$
\frac{\sqrt{t}}{\left(x^{2}+t^{2}\right)^{1 / 4}} \leq 1 \text {. }
$$

Therefore, using the notation of Lemma 4.3, the bound (15) becomes

$$
\sqrt{t} \cdot e^{-t} I_{x}(t) \leq \exp \left(h_{x}(t)\right) \leq\left(\frac{t}{x+t}\right)^{x / 2}
$$

which proves the claim, again provided that $x \geq 2$. If $x=0$, the claim follows from Lemma 4.1 as well as Remark 4.2, which shows that the constant $C$ in Lemma 4.1 satisfies $C \leq 1$. It remains to consider the case when $x=1$.

The series representation $(13)$ of $I_{x}(t)$ gives

$$
\begin{aligned}
0 & \leq I_{1}(t) \leq(t / 2) \sum_{n=0}^{\infty} \frac{(t / 2)^{2 n}}{n ! \Gamma(n+2)} \\
& \leq(t / 2)\left(\sum_{n=0}^{\infty} \frac{(t / 2)^{n}}{n !}\right)\left(\sum_{n=0}^{\infty} \frac{(t / 2)^{n}}{\Gamma(n+2)}\right) \leq(t / 2) e^{t} .
\end{aligned}
$$

For $t \leq 1, t / 2 \leq 1 / \sqrt{(t+1)}$, so then

$$
\sqrt{t} \cdot e^{-t} I_{1}(t) \leq \sqrt{t} \cdot(t / 2) \leq\left(\frac{t}{t+1}\right)^{1 / 2}, \quad \text { for } t \leq 1 .
$$


From (14), we have that $I_{1}(t) \leq I_{0}(t)$ since $\cos (x \theta) \leq 1$. With this, Lemma 4.1 implies

$$
e^{-t} I_{1}(t) \leq e^{-t} I_{0}(t) \leq C / \sqrt{t}
$$

In Remark 4.2, it was argued that $C<1 / \sqrt{2}$. With this, we have for $t \geq 1$ the inequalities

$$
C / \sqrt{t} \leq 1 / \sqrt{2 t} \leq 1 / \sqrt{(t+1)}
$$

so then

$$
e^{-t} I_{1}(t) \leq 1 / \sqrt{(t+1)}, \quad \text { for } t \geq 1
$$

Combining (33) and (34), we have the claimed assertion for $x=1$, which completes the proof for all integers $x \geq 0$.

REMARK 4.5. To be precise, the bound in Corollary 4.4 in the case $x=1$ is not needed in this article. We included the statement and proof for the sake of completeness.

LEMMA 4.6. Fix $t \geq 0$ and nonnegative integers $x$ and $n_{0}$. Then for all $n \geq n_{0}$, we have the uniform bound

$$
0 \leq \sqrt{\left(n^{2} t\right)} \cdot e^{-n^{2} t} I_{n x}\left(n^{2} t\right) \leq\left(\frac{n_{0} t}{x+n_{0} t}\right)^{n_{0} x / 2}=\left(1+\frac{x}{n_{0} t}\right)^{-n_{0} x / 2} .
$$

Proof. The positive lower bound is obvious from the series definition of the $I$-Bessel function, so we focus on proving the upper bound. From Corollary 4.4 , we have

$$
\sqrt{\left(n^{2} t\right)} \cdot e^{-n^{2} t} I_{n x}\left(n^{2} t\right) \leq\left(\frac{n^{2} t}{n x+n^{2} t}\right)^{n x / 2}
$$

Elementary algebra yields

$$
\left(\frac{n^{2} t}{n x+n^{2} t}\right)^{-1}=1+\frac{x}{n t}=1+\frac{x^{2} /(2 t)}{(n x / 2)},
$$

so then we have

$$
\sqrt{\left(n^{2} t\right)} \cdot e^{-n^{2} t} I_{n x}\left(n^{2} t\right) \leq\left(\frac{n^{2} t}{n x+n^{2} t}\right)^{n x / 2}=\left(1+\frac{x^{2} /(2 t)}{(n x / 2)}\right)^{-n x / 2} .
$$


For any constant $c>0$, consider the function

$$
g(y)=\left(1+\frac{c}{y}\right)^{y} .
$$

We claim that $g(y)$ is monotone increasing in $y$. Indeed, using logarithmic differentiation, we have that

$$
\begin{aligned}
\frac{g^{\prime}(y)}{g(y)} & =\log \left(1+\frac{c}{y}\right)+y \cdot \frac{1}{1+c / y} \cdot \frac{-c}{y^{2}} \\
& =\log \left(1+\frac{c}{y}\right)-\frac{c}{c+y}=\log \left(\frac{c+y}{y}\right)-\frac{c}{c+y} \\
& =-\log \left(\frac{y}{c+y}\right)-\frac{c}{c+y}=-\log \left(1-\frac{c}{c+y}\right)-\frac{c}{c+y}
\end{aligned}
$$

Clearly, if $0 \leq u<1$, the function $-\log (1-u)-u$ is positive. Since $g(y)>0$, we conclude that $g^{\prime}(y)>0$, which shows that $g(y)$ is monotone increasing in $y$. By taking $c=x^{2} /(2 t)$ and $y=n x / 2$, we have, for $n \geq n_{0}$, the inequality

$$
\left(1+\frac{x^{2} /(2 t)}{\left(n_{0} x / 2\right)}\right)^{n_{0} x / 2} \leq\left(1+\frac{x^{2} /(2 t)}{(n x / 2)}\right)^{n x / 2}
$$

which gives

$$
\begin{aligned}
\left(1+\frac{x^{2} /(2 t)}{(n x / 2)}\right)^{-n x / 2} & \leq\left(1+\frac{x^{2} /(2 t)}{\left(n_{0} x / 2\right)}\right)^{-n_{0} x / 2} \\
& =\left(1+\frac{x}{n_{0} t}\right)^{-n_{0} x / 2}=\left(\frac{n_{0} t}{n_{0} t+x}\right)^{n_{0} x / 2} .
\end{aligned}
$$

By substituting (36) in (35), the lemma is proved.

Proposition 4.7. Let $n(u)$ be a sequence of positive integers parametrized by $u \in \mathbf{Z}^{+}$such that $n(u) / u \rightarrow \alpha>0$ as $u \rightarrow \infty$. Then for any $t>0$ and nonnegative integer $k \geq 0$, we have

$$
\lim _{u \rightarrow \infty} n(u) \cdot e^{-2 u^{2} t} I_{n(u) k}\left(2 u^{2} t\right)=\frac{\alpha}{\sqrt{4 \pi t}} \cdot e^{-(\alpha k)^{2} /(4 t)} .
$$

Proof. We work with the integral expression for the $I$-Bessel function given in Section 2.2, namely, that for any integer $x$, we have

$$
I_{x}(t)=\frac{1}{\pi} \int_{0}^{\pi} e^{t \cos (\theta)} \cos (\theta x) d \theta .
$$


To begin, assume $k>0$. If we let $y=n(u) k \theta$, then we can write

$$
\begin{aligned}
n(u) e^{-2 u^{2} t} I_{n(u) k}\left(2 u^{2} t\right) & =\frac{n(u)}{\pi} \int_{0}^{\pi} e^{-2 u^{2} t(1-\cos (\theta))} \cos (\theta n(u) k) d \theta \\
& =\frac{1}{k \pi} \int_{0}^{n(u) k \pi} e^{-2 u^{2} t(1-\cos (y /(n(u) k)))} \cos (y) d y .
\end{aligned}
$$

For all $v \in[0, \pi]$, one can easily show that

$$
\frac{1}{2}-\frac{\pi^{2}}{24} \leq \frac{1}{2}-\frac{v^{2}}{24} \leq \frac{1-\cos (v)}{v^{2}} .
$$

Let $c=1 / 2-\pi^{2} / 24$, which, numerically, can be shown to satisfy $c>0$. Setting $v=y /(n(u) x)$, we get for $y \in[0, u x \pi]$ the uniform bound

$$
\frac{1-\cos (y /(n(u) k))}{1 / u^{2}} \geq c\left(\frac{y u}{n(u) k}\right)^{2} .
$$

In addition, observe that

$$
\lim _{u \rightarrow \infty} \frac{1-\cos (y /(n(u) k))}{1 / u^{2}}=\frac{1}{2}\left(\frac{y}{\alpha k}\right)^{2} .
$$

Choose $u_{0}$ such that for $u>u_{0}$ we have $\alpha / 2<n(u) / u<2 \alpha$. Using elementary bounds, we have for any $u>u_{0}$ the inequalities

$$
\begin{aligned}
\left|n(u) e^{-2 u^{2} t} I_{n(u) k}\left(2 u^{2} t\right)\right| & \leq \frac{1}{k \pi} \int_{0}^{n(u) k \pi} e^{-c y^{2} t /(2 \alpha k)^{2}} d y \\
& \leq \frac{1}{k \pi} \int_{0}^{\infty} e^{-c y^{2} t /(2 \alpha k)^{2}} d y=\frac{\alpha}{\sqrt{\pi c t}} .
\end{aligned}
$$

Therefore, by the Lebesgue dominated convergence theorem, we have

$$
\begin{aligned}
\lim _{u \rightarrow \infty} n(u) \cdot e^{-2 u^{2} t} I_{n(u) k}\left(2 u^{2} t\right) & =\frac{1}{k \pi} \int_{0}^{\infty} e^{-y^{2} t /(\alpha k)^{2}} \cos (y) d y \\
& =\frac{\alpha}{\sqrt{4 \pi t}} \cdot e^{-(\alpha k)^{2} /(4 t)} .
\end{aligned}
$$

If $x=0$, the proof follows a similar pattern using instead the substitution $y=\theta u$; for the sake of brevity, we omit the details.

Remark 4.8. Proposition 4.7 was proved by Athreya [1, Theorem 2] using a certain local central limit theorem. For convenience we included a quick self-contained proof. 


\section{$\S 5$. Asymptotic behavior of spectral determinants}

In this section we use the bounds and asymptotic relations from Section 4 to prove our main theorem, namely, the asymptotic behavior of the spectral determinant associated to a sequence of degenerating discrete tori. We begin by proving that the associated family of traces of heat kernels converges pointwise through degeneration (Proposition 5.2) and then prove uniform bounds for heat traces for long time (Proposition 5.4) and small time (Proposition 5.5 and Lemma 5.6). After these results, we analyze the expression for $\mathcal{H}_{N}(0)$ from Theorem 3.6, namely,

$$
\mathcal{H}_{N}(0)=-\int_{0}^{\infty}\left(\theta_{N}(t)-V(N) e^{-2 d t} I_{0}(2 t)^{d}-1+e^{-t}\right) \frac{d t}{t} .
$$

Ultimately we compare the limiting value of (37) through degeneration with the expression for the spectral determinant on the real torus, which is stated in (21) in Section 2.6. These computations are given in the proof of Theorem 5.8, which is the main result of this section.

\subsection{Degenerating sequences of discrete tori}

We consider $d$-tuples of integers $N(u)$ parametrized by a positive integer $u$ in such a way that

$$
\frac{1}{u} N(u)=\frac{1}{u}\left(n_{1}, \ldots, n_{d}\right) \rightarrow\left(\alpha_{1}, \ldots, \alpha_{d}\right), \text { as } u \rightarrow \infty .
$$

Let $A$ be the diagonal matrix with the $\alpha_{j}$ on the diagonal. Recall the notation $V(N)=n_{1}, \ldots, n_{d}$ and $V(A)=\alpha_{1}, \ldots, \alpha_{d}$. From $(38), V(N(u)) / u^{d} \rightarrow$ $V(A)$ when $u \rightarrow \infty$. For the sake of brevity, and for this section, we assume that $V(A)>0$, meaning the limiting real torus $A \mathbf{Z}^{d} \backslash \mathbf{R}^{d}$ has dimension $d$. Using the elementary change of variables $t \mapsto u^{2} t$, we write (37) as

$$
\begin{aligned}
& \mathcal{H}_{N(u)}(0) \\
& \quad=-\int_{0}^{\infty}\left(\theta_{N(u)}\left(u^{2} t\right)-V(N(u))\left(e^{-2 u^{2} t} I_{0}\left(2 u^{2} t\right)\right)^{d}-1+e^{-u^{2} t}\right) \frac{d t}{t},
\end{aligned}
$$

which is the form that we will study. The results of this section are designed to determine the asymptotic behavior of (39) as $u \rightarrow \infty$.

Proposition 5.2. For each fixed $t>0$, we have the pointwise limit

$$
\theta_{N(u)}\left(u^{2} t\right) \rightarrow \Theta_{A}(t), \quad \text { as } u \rightarrow \infty
$$


In other words, the rescaled theta functions on the discrete tori approach the theta function on the limiting real torus $A \mathbf{Z}^{d} \backslash \mathbf{R}^{d}$.

Proof. We begin by writing the theta function using its expansion involving $I$-Bessel functions, namely,

$$
\theta_{N(u)}\left(u^{2} t\right)=\sum_{k_{1}, \ldots, k_{d}=-\infty}^{\infty} \prod_{j=1}^{d} n_{j}(u) e^{-2 u^{2} t} I_{n_{j}(u) k_{j}}\left(2 u^{2} t\right) .
$$

From Proposition 4.7, we have, for any $t>0$, the pointwise limit

$$
\prod_{j=1}^{d} n_{j}(u) e^{-2 u^{2} t} I_{n_{j}(u) k_{j}}\left(2 u^{2} t\right) \rightarrow V(A) \prod_{j=1}^{d} \frac{1}{\sqrt{(4 \pi t)}} e^{-\left(\alpha_{j} k_{j}\right)^{2} /(4 t)}, \quad \text { as } u \rightarrow \infty .
$$

Since

$$
\Theta_{A}(t)=V(A) \sum_{k_{1}, \ldots, k_{d}=-\infty}^{\infty} \prod_{j=1}^{d} \frac{1}{\sqrt{(4 \pi t)}} e^{-\left(\alpha_{j} k_{j}\right)^{2} /(4 t)},
$$

the result will follow if we can interchange the limit in $u$ with the infinite sum in (40).

Choose $u_{0}$ such that for each $j=1, \ldots, d, \alpha_{j} / 2<n_{j}(u) / u<2 \alpha_{j}$ for all $u>u_{0}$. We can rewrite (40) into a sum of $d+1$ subseries, each determined by the number of $k_{j}$ which are equal to zero. Fix some $u_{0} \geq 0$ sufficiently large. Then from Lemmas 4.1 and 4.6, we have that the subseries consisting of terms with exactly $r$ of the $k_{j}$ equal to zero is bounded from above for any $u>u_{0}$ by

$$
\begin{aligned}
& 2^{r} C^{r}(2 t)^{-r / 2} V(A) \sum_{k_{1}, \ldots, k_{r}=1}^{\infty} \prod_{j=1}^{r}\left(1+\frac{\alpha_{j} k_{j}}{4 u_{0} t}\right)^{-u_{0} \alpha_{j} k_{j} / 2} \\
& \leq 2^{r} C^{r}(2 t)^{-r / 2} V(A) \sum_{k_{1}, \ldots, k_{r}=1}^{\infty} \prod_{j=1}^{r} r_{j}^{k_{j}}
\end{aligned}
$$

where

$$
r_{j}=\left(1+\frac{\alpha_{j}}{4 u_{0} t}\right)^{-u_{0} \alpha_{j} / 2}<1
$$

Obviously,

$$
\sum_{k_{1}, \ldots, k_{r}=1}^{\infty} \prod_{j=1}^{r} r_{j}^{k_{j}}=\prod_{j=1}^{r}\left(\frac{r_{j}}{1-r_{j}}\right)
$$


Therefore, the series in (40) is uniformly convergent for fixed $t$, so we can interchange the limit in $u$ and the summation in (40), which completes the proof.

LEMMA 5.3. Let

$$
\theta_{\mathrm{abs}}(t)=2 \sum_{j=1}^{\infty} e^{-c j^{2} t}
$$

with $c=4 \pi^{2}\left(1-\pi^{2} / 24\right)^{2}$. Assume $u_{0}$ is such that $\alpha_{k} / 2<n_{k} / u<2 \alpha_{k}$ for all $k=1, \ldots, d$ and $u>u_{0}$. Then for any $t>0$ and $u>u_{0}$, we have the bound

$$
\theta_{N(u)}\left(u^{2} t\right) \leq \prod_{k=1}^{d}\left(1+e^{-4 u_{0}^{2} t}+\theta_{\mathrm{abs}}\left(\alpha_{k} t / 2\right)\right) .
$$

Proof. Recall from [15] that

$$
\theta_{n_{k}(u)}(t)=1+e^{-4 t}+2 \sum_{j=1}^{n_{k}(u) / 2-1} e^{-4\left(\sin \left(\pi j / n_{k}(u)\right)\right)^{2} t}
$$

if $n_{k}(u)$ is even, and

$$
\theta_{n_{k}(u)}(t)=1+2 \sum_{j=1}^{\left(n_{k}(u)-1\right) / 2} e^{-4\left(\sin \left(\pi j / n_{k}(u)\right)\right)^{2} t}
$$

if $n_{k}(u)$ is odd. In either case, we will use the elementary bound

$$
\sin x \geq x-x^{3} / 6, \quad \text { for } x \in[0, \pi / 2] .
$$

To prove this inequality, one considers the Taylor series expansion with error at $x=0$ for $\sin (x)$, together with the observation that the error term is positive. Taking $x=\pi j / n_{k}(u)$, we then have that

$$
n_{k}(u) \sin \left(\pi j / n_{k}(u)\right) \geq \pi j\left(1-(\pi j)^{2} /\left(6 n_{k}(u)^{2}\right)\right) .
$$

We may assume that $j \leq n_{k}(u) / 2$, so then $j / n_{k}(u) \leq 1 / 2$; hence

$$
n_{k}(u) \sin \left(\pi j / n_{k}(u)\right) \geq \pi j\left(1-\pi^{2} / 24\right) .
$$

It is important to use the trivial bound $1-\pi^{2} / 24>0$. With all this, we arrive at the inequality

$$
\theta_{n_{j}(u)}\left(u^{2} t\right) \leq 1+e^{-4 u^{2} t}+2 \sum_{j=1}^{n / 2} e^{-c \alpha_{k} j^{2} t / 2} \leq 1+e^{-4 u_{0}^{2} t}+2 \sum_{j=1}^{\infty} e^{-c \alpha_{k} j^{2} t / 2},
$$


with $c=4 \pi^{2}\left(1-\pi^{2} / 24\right)^{2}>0$. Therefore, we have shown that for any $u>u_{0}$, we have the bound

$$
\theta_{n}\left(u^{2} t\right) \leq 1+e^{-4 u_{0}^{2} t}+\theta_{\mathrm{abs}}(t)
$$

Since

$$
\theta_{N(u)}\left(u^{2} t\right)=\prod_{j=1}^{d} \theta_{n_{k}(u)}\left(u^{2} t\right),
$$

the proof of the lemma is complete.

Proposition 5.4. With notation as above, we have that

$$
\begin{aligned}
\int_{1}^{\infty} & \left(\theta_{N(u)}\left(u^{2} t\right)-V(N(u))\left(e^{-2 u^{2} t} I_{0}\left(2 u^{2} t\right)\right)^{d}-1+e^{-u^{2} t}\right) \frac{d t}{t} \\
= & \int_{1}^{\infty}\left(\Theta_{A}(t)-1\right) \frac{d t}{t}-\frac{2}{d} V(A)(4 \pi)^{-d / 2}+o(1), \quad \text { as } u \rightarrow \infty
\end{aligned}
$$

Proof. Write

$$
\begin{aligned}
\int_{1}^{\infty} & \left(\theta_{N(u)}\left(u^{2} t\right)-V(N(u))\left(e^{-2 u^{2} t} I_{0}\left(2 u^{2} t\right)\right)^{d}-1+e^{-u^{2} t}\right) \frac{d t}{t} \\
= & \int_{1}^{\infty}\left(\theta_{N(u)}\left(u^{2} t\right)-1\right) \frac{d t}{t}-\int_{1}^{\infty} V(N(u))\left(e^{-2 u^{2} t} I_{0}\left(2 u^{2} t\right)\right)^{d} \frac{d t}{t} \\
& +\int_{1}^{\infty} e^{-u^{2} t} \frac{d t}{t}
\end{aligned}
$$

and consider the three integrals separately. Trivially, we have

$$
\int_{1}^{\infty} e^{-u^{2} t} \frac{d t}{t} \rightarrow 0, \quad \text { as } u \rightarrow \infty .
$$

Next, we claim that

$$
\begin{aligned}
V(N) \int_{1}^{\infty}\left(e^{-2 u^{2} t} I_{0}\left(2 u^{2} t\right)\right)^{d} \frac{d t}{t} & \rightarrow V(A) \int_{1}^{\infty}(4 \pi t)^{-d / 2} \frac{d t}{t} \\
& =\frac{2}{d} V(A)(4 \pi)^{-d / 2}, \quad \text { as } u \rightarrow \infty .
\end{aligned}
$$

Indeed, the pointwise convergence of the integrands in (44) is proved in Proposition 4.7, and the uniform bound from Lemma 4.1, which is integrable 
on $(1, \infty)$ with respect to the measure $d t / t$, allows one to apply the Lebesgue dominated convergence theorem to prove the claim. It remains to show that

$$
\int_{1}^{\infty}\left(\theta_{N(u)}\left(u^{2} t\right)-1\right) \frac{d t}{t} \rightarrow \int_{1}^{\infty}\left(\Theta_{A}(t)-1\right) \frac{d t}{t}, \quad \text { as } u \rightarrow \infty .
$$

The pointwise convergence of the integrands is proved in Proposition 5.2, and Lemma 5.3 establishes a uniform, integrable upper bound so that, again, we may apply the Lebesgue dominated convergence theorem, thus completing the proof.

Proposition 5.5. With notation as above, we have that

$$
\begin{gathered}
\int_{0}^{1}\left(\theta_{N(u)}\left(u^{2} t\right)-V(N)\left(e^{-2 u^{2} t} I_{0}\left(2 u^{2} t\right)\right)^{d}\right) \frac{d t}{t} \\
\rightarrow \int_{0}^{1}\left(\Theta_{A}(t)-V(A)(4 \pi t)^{-d / 2}\right) \frac{d t}{t}
\end{gathered}
$$

as $u \rightarrow \infty$.

Proof. For fixed $t<1$, we have the pointwise convergence $\theta_{N(u)}\left(u^{2} t\right)-V(N)\left(e^{-2 u^{2} t} I_{0}\left(2 u^{2} t\right)\right)^{d} \rightarrow \Theta_{A}(t)-V(A)(4 \pi t)^{-d / 2}, \quad$ as $u \rightarrow \infty$, directly from Propositions 4.7 and 5.2. It remains to show that we have uniform, integrable bounds for the integrand so then the proposition will follow from the Lebesgue dominated convergence theorem. For this, we write

$$
\begin{aligned}
& \theta_{N(u)}\left(u^{2} t\right)-V(N(u))\left(e^{-2 u^{2} t} I_{0}\left(2 u^{2} t\right)\right)^{d} \\
& \quad=V(A) \sum_{K \neq 0} \prod_{k_{1}, \ldots, k_{d}} u e^{-2 u^{2} t} I_{n_{j}(u) k_{j}}\left(2 u^{2} t\right) .
\end{aligned}
$$

The bounds (41) and (42) apply. Since each $n_{j}(u)$ tends to infinity, one can choose $u_{0}$ such that for each $j=1, \ldots, d$ and $u>u_{0}$, we have the inequality $n_{j}(u) k_{j}>d+2$, which implies that the upper bound in (42) is integrable on $(0,1)$ with respect to $d t / t$.

Lemma 5.6. For $u \in \mathbf{R}$, we have the asymptotic formula

$$
\int_{0}^{1}\left(e^{-u^{2} t}-1\right) \frac{d t}{t}=\Gamma^{\prime}(1)-\log \left(u^{2}\right)+o(1), \quad \text { as } u \rightarrow \infty .
$$


Proof. Choose $\varepsilon \in(0,1)$. By employing integration by parts, we can write

$$
\begin{aligned}
\int_{\varepsilon}^{1}\left(e^{-u^{2} t}-1\right) \frac{d t}{t} & =\int_{\varepsilon}^{1}\left(e^{-u^{2} t}-1\right) d \log (t) \\
& =\left.\log (t) \cdot\left(e^{-u^{2} t}-1\right)\right|_{t=\varepsilon} ^{1}+u^{2} \int_{\varepsilon}^{1} \log (t) e^{-u^{2} t} d t \\
& =\log (\varepsilon) \cdot\left(1-e^{-u^{2} \varepsilon}\right)+u^{2} \int_{\varepsilon}^{1} \log (t) e^{-u^{2} t} d t
\end{aligned}
$$

For fixed $u$, we have that $1-e^{-u^{2} \varepsilon}=O(\varepsilon)$ as $\varepsilon$ approaches zero, so then

$$
\int_{0}^{1}\left(e^{-u^{2} t}-1\right) \frac{d t}{t}=u^{2} \int_{0}^{1} \log (t) e^{-u^{2} t} d t
$$

If we let $v=u^{2} t$, then $d v=u^{2} d t$; hence

$$
\begin{aligned}
\int_{0}^{1}\left(e^{-u^{2} t}-1\right) \frac{d t}{t} & =\int_{0}^{u^{2}} \log \left(v / u^{2}\right) e^{-v} d v \\
& =\int_{0}^{u^{2}} \log (v) e^{-v} d v-\log \left(u^{2}\right) \int_{0}^{u^{2}} e^{-v} d v .
\end{aligned}
$$

By definition

$$
\Gamma(s)=\int_{0}^{\infty} e^{-v} v^{s} \frac{d v}{v},
$$

which implies that

$$
\Gamma^{\prime}(1)=\int_{0}^{\infty} e^{-v} \log (v) d v=\int_{0}^{u^{2}} e^{-v} \log (v) d v+\int_{u^{2}}^{\infty} e^{-v} \log (v) d v,
$$

thus yielding the relation

$$
\begin{aligned}
\int_{0}^{1}\left(e^{-u^{2} t}-1\right) \frac{d t}{t} & =\Gamma^{\prime}(1)-\int_{u^{2}}^{\infty} e^{-v} \log (v) d v-\log \left(u^{2}\right) \int_{0}^{u^{2}} e^{-v} d v \\
& =\Gamma^{\prime}(1)-\log \left(u^{2}\right)+\log \left(u^{2}\right) e^{-u^{2}}-\int_{u^{2}}^{\infty} \log (v) e^{-v} d v
\end{aligned}
$$

For $x>1$, we have the trivial inequalities $0 \leq \log x \leq 2 e^{x / 2}$, so then

$$
0 \leq \int_{u^{2}}^{\infty} \log (v) e^{-v} d v \leq 2 \int_{u^{2}}^{\infty} e^{-v / 2} d v=4 e^{-u^{2} / 2}=o(1), \quad \text { as } u \rightarrow \infty .
$$

Trivially, $\log \left(u^{2}\right) e^{-u^{2}}=o(1)$ as $u \rightarrow \infty$. By substituting this estimate and (47) into (46), the proof of the lemma is complete. 
Corollary 5.7. With notation as above, we have

$$
\begin{aligned}
& \int_{0}^{1}\left(\theta_{N(u)}\left(u^{2} t\right)-V(N)\left(e^{-2 u^{2} t} I_{0}\left(2 u^{2} t\right)\right)^{d}-1+e^{-u^{2} t}\right) \frac{d t}{t} \\
& \quad \rightarrow \int_{0}^{1}\left(\Theta_{A}(t)-V(A)(4 \pi t)^{-d / 2}\right) \frac{d t}{t}+\Gamma^{\prime}(1)-\log \left(u^{2}\right)+o(1), \quad \text { as } u \rightarrow \infty .
\end{aligned}
$$

Proof. Simply combine Proposition 5.5 and Lemma 5.6.

THEOREM 5.8. With notation as above, we have

$$
\mathcal{H}_{N(u)}(0)=\log u^{2}-\zeta_{A}^{\prime}(0)+o(1), \quad \text { as } u \rightarrow \infty .
$$

Equivalently, we have

$$
\log \left(\prod_{\Lambda_{j} \neq 0} \Lambda_{j}\right)=V(N(u)) \mathcal{I}_{d}(0)+\log u^{2}-\zeta_{A}^{\prime}(0)+o(1), \quad \text { as } u \rightarrow \infty,
$$

where

$$
\mathcal{I}_{d}(0)=-\int_{0}^{\infty}\left(e^{-2 d t} I_{0}(2 t)^{d}-e^{-t}\right) \frac{d t}{t} .
$$

Proof. To begin, combine Proposition 5.4 and Corollary 5.7 to get the asymptotic expansion of $V(N(u)) \mathcal{H}_{N(u)}(0)$ out to $o(1)$. Then combine the terms in the expansion with the expression in (21) to complete the proof of the first assertion. One then substitutes the first assertion into the identity stated in Theorem 3.6 to prove the second relation.

REMARK 5.9. Using the notation of spectral determinants, one can rewrite the main result in Theorem 5.8 as stating the asymptotic relation

$$
\begin{aligned}
\log \operatorname{det}^{*} \boldsymbol{\Delta}_{D T, N(u)}= & V(N(u)) \mathcal{I}_{d}(0)+\log u^{2} \\
& +\log \operatorname{det}^{*} \boldsymbol{\Delta}_{\mathrm{RT}, A}+o(1), \quad \text { as } u \rightarrow \infty .
\end{aligned}
$$

In words, the asymptotic expansion of the log-determinant of the Laplacian on the discrete torus $D T_{N(u)}$ has a lead term

$$
\begin{aligned}
& V(N(u)) \mathcal{I}_{d}(0)+\log u^{2} \\
& \quad=-V(N(u)) \int_{0}^{\infty}\left(e^{-2 d t}\left(I_{0}(2 t)\right)^{d}-e^{-t}\right) \frac{d t}{t}+\log u^{2} \\
& \quad=V(N(u))\left(\log 2 d-\int_{0}^{\infty} e^{-d t}\left(I_{0}(t)^{d}-1\right) \frac{d t}{t}\right)+\log u^{2},
\end{aligned}
$$

with a constant term equal to the log-determinant of the Laplacian on the real torus $A \mathbf{Z}^{d} \backslash \mathbf{R}^{d}$. 
REMARK 5.10. As previously discussed, two zeta functions are being considered: the spectral zeta function $\zeta_{A}$ associated to the real torus $A \mathbf{Z}^{d} \backslash \mathbf{R}^{d}$ and the Epstein zeta function $Z(\cdot, Q)$ associated to the positive definite $d \times d$ matrix $Q$. Classical and elementary mathematical considerations show that $Z(\cdot, Q)=\zeta_{A}$, where $Q$ is the form associated to $A^{*}$, the dual lattice of $A$. This remark needs to be kept in mind when reading the main result in Theorem 5.8 as restated in Remark 5.9.

\section{§6. Example: cases $d=1$ and $d=2$}

As stated in Section 1, the lead term in the asymptotic of $\log \operatorname{det}^{*} \boldsymbol{\Delta}_{D T, N(u)}$ for $d=2$ was first computed by Kasteleyn [16]. Duplantier and David [9] expressed the next-order term in terms of the Dedekind $\eta$ function. In this section we rederive these results by the methods introduced in this article and go further by indicating how to obtain the complete asymptotic expansion of $\log \operatorname{det}^{*} \boldsymbol{\Delta}_{D T, N(u)}$ when $d=2$. We begin by making explicit the case $d=1$.

\subsection{The case $d=1$}

Known evaluations of the Riemann zeta function $\zeta_{\mathbf{Q}}$ at $s=0$ yield the relations $\zeta_{\mathbf{Q}}(0)=-1 / 2$ and $\zeta_{\mathbf{Q}}^{\prime}(0)=-(1 / 2) \log (2 \pi)$. Let $N(u)=n$ with $n / u \rightarrow \alpha>0$. With all this, we can write

$$
\log n^{2}=\log u^{2}+4 \log (2 \pi / \alpha) \zeta_{\mathbf{Q}}(0)-4 \zeta_{\mathbf{Q}}^{\prime}(0)+o(1), \quad \text { as } u \rightarrow \infty
$$

We claim that (48) is equivalent to the main theorem when $d=1$. First, it is obvious that the number of spanning trees in $n \mathbf{Z} \backslash \mathbf{Z}$ is $n$ and, hence, that the determinant of the combinatorial Laplacian is $n^{2}$, so the left-hand side of (5) is $\log n^{2}$. Regarding the right-hand side, it is possible to directly verify through the Mellin transform that

$$
\int_{0}^{\infty} e^{-2 t}\left(I_{0}(2 t)-1\right) \frac{d t}{t}=\log 2,
$$

which implies, in our notation, that $\mathcal{I}_{1}(0)=0$. Hence, the lead term in the asymptotic expansion (5) when $d=1$ is zero. The continuous Laplacian on $n \mathbf{Z} \backslash \mathbf{R}$ has the set of eigenvalues given by $\left\{(2 \pi)^{2}(n / \alpha)^{2}\right\}$ for $n \in \mathbf{Z}$, so the spectral zeta function is

$$
\zeta_{\alpha}(s)=2(2 \pi / \alpha)^{-2 s} \zeta_{\mathbf{Q}}(2 s)
$$


thus

$$
\zeta_{\alpha}^{\prime}(0)=-\log \operatorname{det}^{*} \boldsymbol{\Delta}_{\mathrm{RT}, \alpha}=-4 \log (2 \pi / \alpha) \zeta_{\mathbf{Q}}(0)+4 \zeta_{\mathbf{Q}}^{\prime}(0),
$$

which agrees with (48) and confirms, by direct computation, the main theorem when $d=1$.

\subsection{The lead term when $d=2$}

We will evaluate the expression

$$
\mathcal{I}_{d}(0)=\log (2 d)-\int_{0}^{\infty} e^{-d t}\left(I_{0}(t)^{d}-1\right) \frac{d t}{t}
$$

when $d=2$. Recall from Section 2.8 the Mellin inversion formula, (25). Let us set $x=y=0$ and move the contour of integration to the line $\operatorname{Re}(\sigma)=-1 / 4$. The integrand has a pole at $z=0$, which results in the expression

$$
\begin{aligned}
\widetilde{f_{0} f_{0}}(s)= & \frac{1}{2 \pi i} \int_{\operatorname{Re}(z)=(-1 / 4)} \frac{\Gamma(s-z) \Gamma(1 / 2+z-s) \Gamma(z) \Gamma(1 / 2-z)}{\pi \Gamma(1+z-s) \Gamma(1-z)} d z \\
& +\frac{\Gamma(s) \Gamma(1 / 2-s) \Gamma(1 / 2)}{\pi \Gamma(1-s) \Gamma(1)},
\end{aligned}
$$

so then, using the definition of $\Gamma(s)$ as the Mellin transform of $e^{-t}$, we have

$$
\begin{aligned}
\widetilde{f_{0} f_{0}}(s)-\Gamma(s)= & \int_{0}^{\infty} e^{-t}\left[I_{0}(t / 2)^{2}-1\right] t^{s} \frac{d t}{t} \\
= & \frac{1}{2 \pi i} \int_{\operatorname{Re}(z)=(-1 / 4)} \frac{\Gamma(s-z) \Gamma(1 / 2+z-s) \Gamma(z) \Gamma(1 / 2-z)}{\pi \Gamma(1+z-s) \Gamma(1-z)} d z \\
& +\frac{\Gamma(s) \Gamma(1 / 2-s)}{\sqrt{\pi} \Gamma(1-s)}-\Gamma(s) .
\end{aligned}
$$

By Taylor's theorem, we can write

$$
\lim _{s \rightarrow 0}\left(\frac{\Gamma(s) \Gamma(1 / 2-s)}{\sqrt{\pi} \Gamma(1-s)}-\Gamma(s)\right)=\frac{-\Gamma^{\prime}(1 / 2)+\sqrt{\pi} \Gamma^{\prime}(1)}{\sqrt{\pi}},
$$

where we have used that $\Gamma(1 / 2)=\sqrt{\pi}$ and $\Gamma(1)=1$. One now uses the relation

$$
\Gamma(z) \Gamma(z+1 / 2)=2^{1-2 z} \sqrt{\pi} \Gamma(2 z),
$$

and by computing the derivative at $z=1 / 2$, one can show that

$$
\frac{-\Gamma^{\prime}(1 / 2)+\sqrt{\pi} \Gamma^{\prime}(1)}{\sqrt{\pi}}=\log 4 .
$$


With these preliminary results, we have shown, by taking $s \rightarrow 0$ in (50), that

$$
\begin{aligned}
& \int_{0}^{\infty} e^{-t}\left[I_{0}(t / 2)^{2}-1\right] \frac{d t}{t} \\
& \quad=\frac{1}{2 \pi i} \int_{\operatorname{Re}(z)=(-1 / 4)} \frac{\Gamma(-z) \Gamma(1 / 2+z) \Gamma(z) \Gamma(1 / 2-z)}{\pi \Gamma(1+z) \Gamma(1-z)} d z+\log 4 \\
& \quad=\log 4-\frac{1}{2 \pi i} \int_{\operatorname{Re}(z)=(-1 / 4)} \frac{1}{z^{2} \cos \pi z} d z
\end{aligned}
$$

where the last equality of integrals comes from employing the identities

$$
\Gamma(x) \Gamma(1-x)=\pi / \sin \pi x, \quad x \Gamma(x)=\Gamma(x+1) .
$$

We now evaluate the last integral in (51) by moving the line of integration toward $\operatorname{Re}(z)=-\infty$. The integrand has poles $z=-n-1 / 2$, for $n=0,1,2, \ldots$, with residues $(-1)^{n}(n+1 / 2)^{-2} / \pi$. Thus

$$
\int_{0}^{\infty} e^{-t}\left[I_{0}(t / 2)^{2}-1\right] t^{s} \frac{d t}{t}=\log 4-\frac{1}{\pi} \sum_{n=0}^{\infty} \frac{(-1)^{n}}{(n+1 / 2)^{2}}=\log 4-\frac{4 G}{\pi}
$$

where $G$ is the Catalan constant

$$
G=\sum_{n=0}^{\infty} \frac{(-1)^{n}}{(2 n+1)^{2}}
$$

With all this, we have shown that

$$
\mathcal{I}_{2}(0)=\frac{4 G}{\pi} .
$$

The relation in (53), which is the main asymptotic term from Theorem 5.8 in the case $d=2$, agrees with the computations from $[9,(3.18)$, page 349$]$ (see also [9, (A.21), page 427]).

\subsection{Secondary terms when $d=2$}

From Theorem 5.8, we have that

$$
\mathcal{H}_{N}(0)=\log u^{2}+\log \operatorname{det}^{*} \boldsymbol{\Delta}_{\mathrm{RT}, d, A}+o(1), \quad \text { as } u \rightarrow 0,
$$

for a degenerating sequence of discrete tori. As before, consider a sequence of integer vectors $N(u)$ for $u \in \mathbf{Z}^{+}$, and assume

$$
\frac{1}{u} N(u)=\frac{1}{u}\left(n_{1}(u), n_{2}(u)\right) \rightarrow\left(\alpha_{1}, \alpha_{2}\right), \quad \text { as } u \rightarrow \infty .
$$


Proposition 4.7 evaluates the heat kernel on $\left(\alpha_{1} \mathbf{Z} \times \alpha_{2} \mathbf{Z}\right) \backslash \mathbf{R}^{2}$, where the Laplacian is

$$
\Delta_{\mathbf{R}^{2}}=-\left(\frac{\partial^{2}}{\partial x_{1}^{2}}+\frac{\partial^{2}}{\partial x_{2}^{2}}\right)
$$

where $x_{1}$ and $x_{2}$ are the usual global coordinates on $\mathbf{R}^{2}$. The action of $\Delta_{\mathbf{R}^{2}}$ on the space of smooth functions on $\left(\alpha_{1} \mathbf{Z} \times \alpha_{2} \mathbf{Z}\right) \backslash \mathbf{R}^{2}$ has the set of eigenvalues given by $\left\{(2 \pi)^{2}\left(\left(n / \alpha_{1}\right)^{2}+\left(m / \alpha_{2}\right)^{2}\right)\right\}$ for $n, m \in \mathbf{Z}$, so then its associated spectral zeta function is

$$
\zeta_{A}(s)=(2 \pi)^{-2 s} \sum_{(n, m) \neq(0,0)} \frac{1}{\left(\left(n / \alpha_{1}\right)^{2}+\left(m / \alpha_{2}\right)^{2}\right)^{s}} .
$$

Let us write

$$
\zeta_{A}(s)=\left(\alpha_{1} \alpha_{2}\right)^{s} E(z, s)
$$

where

$$
E(z, s)=(2 \pi)^{-2 s} \sum_{(n, m) \neq(0,0)} \frac{\operatorname{Im}(z)^{s}}{|n z+m|^{2 s}}, \quad \text { with } z=i\left(\alpha_{2} / \alpha_{1}\right)=i y .
$$

The function $E(z, s)$ admits a meromorphic continuation to all $s \in \mathbf{C}$ with expansion

$$
E(z, s)=-1-s \log \left(y|\eta(z)|^{4}\right)+O(s), \quad \text { as } s \rightarrow 0,
$$

where $z=i\left(\alpha_{2} / \alpha_{1}\right)=i y$ and $\eta(z)$ is the classical Dedekind eta function

$$
\eta(z)=e^{2 \pi i z} \prod_{n=0}^{\infty}\left(1-e^{2 \pi i n z}\right), \quad \text { for any } z \in \mathbf{C} \text { with } \operatorname{Im}(z)>0 .
$$

With all this, we then have that

$$
\log \operatorname{det}^{*} \boldsymbol{\Delta}_{\mathrm{RT}, A}=-\left.\frac{\partial}{\partial s} \zeta_{A}\right|_{s=0}=\log \left(\alpha_{1} \alpha_{2}\right)+\log \left(y|\eta(z)|^{4}\right)
$$

Therefore,

$$
\begin{aligned}
\mathcal{H}_{N}(0) & =\log u^{2}+\log \left(\alpha_{1} \alpha_{2}\right)+\log \left(y|\eta(z)|^{4}\right) \\
& =\log \left(n_{1}(u) n_{2}(u)\right)+\log \left(y|\eta(z)|^{4}\right)
\end{aligned}
$$

Expression (55) agrees with the second-order term given in [9, (A.21), page 427], where those authors used the notation $M=n_{1}(u)$ and $N=n_{2}(u)$. 


\subsection{Error terms when $d=2$}

The asymptotic expansion in Theorem 5.8 contains an error term of $o(1)$. We present here a technique which improves the error term, ultimately obtaining an expansion with error term of arbitrarily small polynomial order, as stated in Section 1. Additionally, the computations we give here offer a second proof of the main theorem in the case $d=2$.

Recall the Mellin inversion formula from Section 2.8, namely,

$$
\begin{aligned}
& \int_{0}^{\infty} I_{x}(t / 2) I_{y}(t / 2) e^{-t} t^{s} \frac{d t}{t} \\
& \quad=\frac{1}{2 \pi i} \int_{\operatorname{Re}(z)=\sigma} \frac{\Gamma(s-z+x) \Gamma(1 / 2+z-s) \Gamma(z+y) \Gamma(1 / 2-z)}{\pi \Gamma(x+1+z-s) \Gamma(y+1-z)} d z
\end{aligned}
$$

For fixed $a$ and $b$, Stirling's formula yields

$$
\frac{\Gamma(z+a)}{\Gamma(z+b)}=z^{a-b}+O\left(z^{a-b-1}\right)
$$

as $z$ tends to infinity anywhere in the half-plane $\operatorname{Re}(z)>\delta>0$. Substituting (57) into (56), we have that the lead term in the asymptotic expansion of $(56)$ is

$$
\begin{aligned}
& \frac{1}{2 \pi i} \int_{\operatorname{Re}(z)=\sigma} x^{s-z-1-z+s} y^{z-1+z} \cdot \frac{1}{\pi} \Gamma(z+1 / 2-s) \Gamma(1 / 2-z) d z \\
& \quad=\frac{x^{2 s}}{\pi \cdot x y} \cdot \frac{1}{2 \pi i} \int_{\operatorname{Re}(z)=\sigma}\left(\frac{y}{x}\right)^{2 z} \Gamma(z+1 / 2-s) \Gamma(1 / 2-z) d z
\end{aligned}
$$

Let us analyze (58) by moving the contour of integration toward $\operatorname{Re}(z)=$ $\infty$. The integrand has poles at $z=1 / 2+n$ with residues equal to $(-1)^{n} / n$ !, so then (58) is equal to

$$
S=\frac{x^{2 s}}{\pi \cdot x y} \cdot \sum_{n=0}^{\infty}\left(\frac{y}{x}\right)^{2 n+1} \frac{(-1)^{n}}{n !} \Gamma(n-s+1) .
$$

Rewrite (59) as

$$
S=\frac{y^{2 s-2}}{\pi} \cdot \sum_{n=0}^{\infty}\left(\frac{y}{x}\right)^{2 n-2 s+2} \frac{(-1)^{n}}{n !} \Gamma(n-s+1),
$$


and use the elementary formula

$$
\Gamma(w) a^{-w}=\int_{0}^{\infty} e^{-a t} t^{w} \frac{d t}{t}
$$

with $w=n-s+1$ and $a=x^{2} / y^{2}$ to get that

$$
S=\frac{y^{2 s-2}}{\pi} \cdot \sum_{n=0}^{\infty} \int_{0}^{\infty} e^{-\left(x^{2} / y^{2}\right) t} t^{n-s+1} \cdot \frac{(-1)^{n}}{n !} \frac{d t}{t} .
$$

Observing that

$$
e^{-t}=\sum_{n=0}^{\infty} t^{n} \cdot \frac{(-1)^{n}}{n !}
$$

we arrive at the relation

$$
S=\frac{y^{2 s-2}}{\pi} \cdot \int_{0}^{\infty} e^{-\left(x^{2} / y^{2}\right) t} e^{-t} t^{-s+1} \frac{d t}{t} .
$$

The integral can be evaluated using (60) with $w=1-s$ and $a=\left(x^{2} / y^{2}+1\right)$, yielding

$$
S=\frac{y^{2 s-2}}{\pi} \cdot \Gamma(1-s)\left(x^{2} / y^{2}+1\right)^{s-1}=\frac{1}{\pi} \Gamma(1-s)\left(x^{2}+y^{2}\right)^{s-1} .
$$

If we set $s=-w$ with $\operatorname{Re}(w)>0$, we then have that

(63) $\int_{0}^{\infty} I_{x}(t / 2) I_{y}(t / 2) e^{-t} t^{-w} \frac{d t}{t}=\frac{1}{\pi} \cdot \frac{\Gamma(1+w)}{\left(x^{2}+y^{2}\right)^{1+w}}+$ lower-order terms.

Finally, we set $x=n j$ and $y=m k$ with fixed $j$ and $k$ and positive integers $n$ and $m$, and we sum over all $n$ and $m$, thus proving that

$$
\begin{aligned}
& \sum_{n, m} \int_{0}^{\infty} I_{n j}(t / 2) I_{m k}(t / 2) e^{-t} t^{-w} \frac{d t}{t} \\
& \quad=\frac{\Gamma(1+w)}{\pi} \sum_{n, m} \frac{1}{\left((n j)^{2}+(m k)^{2}\right)^{1+w}}+\text { lower-order terms. }
\end{aligned}
$$

The series in $(64)$ can be related to the Eisenstein series $E_{A}(z, s)$ with $A=(x, y)$ and $z=i m / n$. One then can use the classical Kronecker's limit formula to evaluate the asymptotic behavior of (64) as $w$ approaches zero. 
In all, the above computations provide another proof of the main theorem when $d=2$.

Stirling's formula can be used to compute further terms in the asymptotic expansion in (57). Specifically, for any positive integers $N$ and $M$, we write

$$
\begin{aligned}
& \frac{\Gamma(x-z) \Gamma(z+y)}{\Gamma(x+1+z) \Gamma(y+1-z)}-\frac{1}{x y}\left(\frac{y}{x}\right)^{2 z} \\
& \quad=\sum_{j, k} P_{j, k}(z) x^{-2 z-1-j} y^{2 z-1-k}+O\left(x^{-2 z-1-N} y^{2 z-1-M}\right)
\end{aligned}
$$

as $x, y \rightarrow \infty$, where $P_{j, k}$ denotes a polynomial in $z$, and the sum is over all integers $j$ and $k$ such that $0 \leq j \leq N$ and $0 \leq k \leq M$, provided $(j, k) \neq(0,0)$. By comparing with (56), we see that the lower-order terms in (64) can be determined by studying

$S_{j, k}(x, y)=\frac{1}{\pi \cdot x^{j+1} y^{k+1}} \cdot \frac{1}{2 \pi i} \int_{\operatorname{Re}(z)=\sigma} P_{j, k}(z)\left(\frac{y}{x}\right)^{2 z} \Gamma(z+1 / 2) \Gamma(1 / 2-z) d z$.

Moving the contour of integration, we get that

$$
S_{j, k}(x, y)=\frac{1}{\pi \cdot x^{j+1} y^{k+1}} \cdot \sum_{n=0}^{\infty}(-1)^{n} P_{j, k}(n+1 / 2)\left(\frac{y}{x}\right)^{2 n+1} .
$$

Since $P_{j, k}(z)$ is a polynomial in $z$, we have that

$$
P_{j, k}(n+1 / 2)\left(\frac{y}{x}\right)^{2 n+1}=P_{j, k}\left(\frac{y}{2} \frac{d}{d y}\right)\left(\frac{y}{x}\right)^{2 n+1},
$$

so then

$$
\begin{aligned}
S_{j, k}(x, y) & =\frac{1}{\pi \cdot x^{j+1} y^{k+1}} \cdot P_{j, k}\left(\frac{y}{2} \frac{d}{d y}\right) \sum_{n=0}^{\infty}(-1)^{n}\left(\frac{y}{x}\right)^{2 n+1} \\
& =\frac{1}{\pi \cdot x^{j+1} y^{k+1}} \cdot P_{j, k}\left(\frac{y}{2} \frac{d}{d y}\right)\left(\frac{x y}{x^{2}+y^{2}}\right)
\end{aligned}
$$

Let us define

$$
B_{j, k}(u)=\sum_{\left(n_{1}, n_{2}\right) \neq(0,0)} S_{j, k}\left(n_{1} u, n_{2} u\right) .
$$

Elementary bounds show that

$$
B_{j, k}(u)=O\left(u^{-j-k-2}\right), \quad \text { when } u \rightarrow \infty, \text { provided }(j, k) \neq(0,0),
$$


since the series in $(67)$ is convergent for $(j, k) \neq(0,0)$. Finally, if we set

$$
F_{n}(u)=\sum_{j+k=n} B_{j, k}(u)
$$

we have that the error term $o(1)$ in Theorem 5.8 can be improved to

$$
\sum_{n=1}^{K} F_{n}(u)+O\left(u^{-K-3}\right), \quad \text { as } u \rightarrow \infty \text { for any integer } K>0 .
$$

To summarize, the coefficients (68) can be explicitly determined from the evaluation (66) which utilizes the precise evaluation of Stirling's formula as stated in (65). The coefficients (68) can be made explicit by evaluation of the polynomials $P_{j, k}(z)$ as defined in (65). We do not pursue this analysis further here but instead leave the details for consideration elsewhere.

\section{§7. Additional considerations}

In this section we present a number of computations and remarks discussing further aspects of the preceding analysis.

\subsection{A precise evaluation of the lead term}

Let

$$
a_{d}=\log (2 d)-\int_{0}^{\infty} e^{-d t}\left(I_{0}(t)^{d}-1\right) \frac{d t}{t}=\log 2-\int_{0}^{\infty}\left(e^{-d t} I_{0}(t)^{d}-e^{-t}\right) \frac{d t}{t}
$$

which is obtained by replacing $2 t$ by $t$ in the integral definition of $\mathcal{I}_{d}(0)$. With this,

$$
a_{d+1}-a_{d}=\int_{0}^{\infty} e^{-d t} I_{0}(t)^{d}\left(1-e^{-t} I_{0}(t)\right) \frac{d t}{t} .
$$

Since $I_{0}(t) \leq e^{t}$, we have that $a_{d+1} \geq a_{d}$. Let us write

$$
e^{-t} I_{0}(t)=1+\sum_{k=1}^{\infty} b_{k} t^{k}
$$

which implies that

$$
a_{d+1}-a_{d}=\sum_{k=1}^{\infty} b_{n} \int_{0}^{\infty} e^{-d t} I_{0}(t)^{d} t^{k} d t
$$


From [15], we have the identity

$$
F(u)=\int_{0}^{\infty} e^{-u t} I_{0}(t) d t=\frac{1}{u \sqrt{u+2}} .
$$

Therefore,

$$
\int_{0}^{\infty} e^{-d t} I_{0}(t)^{d} t^{k} d t=\left(-\partial_{u}\right)^{k} F^{* d}(d)
$$

where $F^{* d}$ denotes the $d$-fold convolution of $F$ with itself, evaluated at $d$. Therefore, we have

$$
a_{d+1}=a_{d}+\sum_{k=1}^{\infty} b_{k} \cdot\left(-\partial_{u}\right)^{k} F^{* d}(d) .
$$

In Section 6.2 we have shown that $a_{2}=4 G / \pi$, where $G$ is the Catalan constant. In addition, one can write

$$
e^{-t} I_{0}(t)=\frac{1}{\pi} \int_{0}^{\pi} e^{-t \sin ^{2}(\theta / 2)} d \theta
$$

so the coefficients $b_{k}$ are explicitly computable. With all this, we have presented another series expansion for $a_{d}$ which could be used to numerically evaluate $a_{d}$.

\subsection{Numerical estimation of the lead term}

This section should be compared with the discussion in Felker and Lyons [11]. By focusing on the lead term in Theorem 5.8, we have that

$$
\begin{aligned}
& \frac{1}{V(N(u))} \sum_{\Lambda_{j}(u) \neq 0} \log \Lambda_{j}(u) \\
& \quad=\log 2 d-\int_{0}^{\infty} e^{-d t}\left(I_{0}(t)^{d}-1\right) \frac{d t}{t}+o(1), \quad \text { as } u \rightarrow \infty .
\end{aligned}
$$

As stated in Section 1, the explicit form of the eigenvalues is such that we recognize the left-hand side of (69) as a Riemann sum, so then (69) implies 
the identity

$$
\begin{aligned}
& \int_{\mathbf{Z}^{d} \backslash \mathbf{R}^{d}} \log \left(2 d-2 \cos \left(2 \pi x_{1}\right)-\cdots-2 \cos \left(2 \pi x_{d}\right)\right) d x_{1} \cdots d x_{d} \\
& =\log 2 d-\int_{0}^{\infty} e^{-d t}\left(I_{0}(t)^{d}-1\right) \frac{d t}{t} .
\end{aligned}
$$

Recall that

$$
I_{0}(t)=1+\sum_{n=1}^{\infty} a_{n} t^{2 n}, \quad \text { where } a_{n}=\frac{1}{2^{2 n}(n !)^{2}}
$$

Let us write

$$
I_{0}(t)^{d}=1+\sum_{n=1}^{\infty} a_{n, d} t^{2 n} .
$$

The coefficients $a_{n, d}$ easily can be written in terms of $a_{n}$ and multinomial coefficients, and (70) can be evaluated as

$$
\begin{aligned}
& \int_{\mathbf{Z}^{d} \backslash \mathbf{R}^{d}} \log \left(2 d-2 \cos \left(2 \pi x_{1}\right)-\cdots-2 \cos \left(2 \pi x_{d}\right)\right) d x_{1} \cdots d x_{d} \\
& \quad=\log 2 d-\sum_{n=1}^{\infty} \frac{a_{n, d} \Gamma(2 n)}{n^{2 d}} .
\end{aligned}
$$

The numerical evaluation of the left-hand side of (70) is difficult for large $d$, both because of the slow growth of the integrand and because the domain of integration involves the $d$-fold product of the unit interval. By comparison, note that the right-hand side of (70) allows for rapid numerical evaluation which improves as $d$ gets larger. Indeed, from Lemma 4.1, we have

$$
\begin{aligned}
0 & \leq \int_{t_{0}}^{\infty} e^{-d t}\left(I_{0}(t)^{d}-1\right) \frac{d t}{t} \leq C^{d} \int_{t_{0}}^{\infty} t^{-d / 2} \frac{d t}{t}+\frac{1}{t_{0}} \int_{t_{0}}^{\infty} e^{-d t} d t \\
& =\frac{2 C^{d} t_{0}^{-d / 2+1}+e^{-d t_{0}}}{d t_{0}} .
\end{aligned}
$$

This bound obviously gets closer to zero as $t_{0}$ grows for any $d$. In addition, the bound decays exponentially in $d$ provided $C t_{0}^{-1 / 2}<1$ which, from the estimate that $C<1 / \sqrt{2}$, implies that we need $t_{0}>1 / 2$. By the mean value theorem, we have, for any $z$ and $a$, the estimate

$$
(x+a)^{d}-x^{d} \leq a d(x+a)^{d-1} .
$$


For any $N$, to be chosen later, we take

$$
x=\sum_{n=0}^{N-1} a_{n} t^{2 n}, \quad a=I_{0}(t)-x<I_{0}(t)
$$

to arrive at the bound

$$
\begin{aligned}
\int_{0}^{t_{0}} e^{-d t}\left(I_{0}(t)^{d}-1\right) \frac{d t}{t} \leq & \int_{0}^{t_{0}} e^{-d t}\left(\left(\sum_{n=0}^{N-1} a_{n} t^{2 n}\right)^{d}-1\right) \frac{d t}{t} \\
& +d \int_{0}^{t_{0}} e^{t}\left(e^{-t} I_{0}(t)\right)^{d-1}\left(\sum_{n=N}^{\infty} a_{n} t^{2 n}\right) \frac{d t}{t} .
\end{aligned}
$$

Choose any $t_{0} \in(1 / 2,1)$. For any $\varepsilon>0$, there is an $N$ such that

$$
\sum_{n=N}^{\infty} a_{n} t^{2 n} \leq \varepsilon t^{2 N}, \quad \text { for } t \in\left[0, t_{0}\right]
$$

Elementary arguments prove the existence of a constant $A>0$ such that

$$
e^{-t} I_{0}(t) \leq 1-A t, \quad \text { for } t \in\left[0, t_{0}\right]
$$

Therefore,

$$
\begin{aligned}
\int_{0}^{t_{0}} e^{t}\left(e^{-t} I_{0}(t)\right)^{d-1}\left(\sum_{n=N}^{\infty} a_{n} t^{2 n}\right) \frac{d t}{t} & \leq \varepsilon d e^{t_{0}} \int_{0}^{t_{0}} t^{2 N-1}(1-A t)^{d-1} d t \\
& \leq \varepsilon e^{t_{0}} t_{0}^{d} / A
\end{aligned}
$$

so then

$$
\begin{aligned}
0 & <\int_{0}^{t_{0}} e^{-d t}\left(I_{0}(t)^{d}-1\right) \frac{d t}{t}-\int_{0}^{t_{0}} e^{-d t}\left(\left(\sum_{n=0}^{N-1} a_{n} t^{2 n}\right)^{d}-1\right) \frac{d t}{t} \\
& \leq \varepsilon e^{t_{0}} t_{0}^{2 N-1} / A .
\end{aligned}
$$

Observe that the upper bound in (72) is independent of $d$. When combining (71) with (72), we conclude that one can estimate

$$
\int_{0}^{\infty} e^{-d t}\left(I_{0}(t)^{d}-1\right) \frac{d t}{t} \quad \text { by } \quad \int_{0}^{t_{0}} e^{-d t}\left(\left(\sum_{n=0}^{N-1} a_{n} t^{2 n}\right)^{d}-1\right) \frac{d t}{t}
$$


with error which is independent of $d$.

It remains to study the asymptotic behavior in $d$ of the second integral in (73). For this, one needs to expand the $d$-fold product of the polynomial integrand and carry out the integration. The integral of each term can then be expressed in terms of the incomplete gamma function, which in turn can be estimated by the gamma function itself. One then would employ elementary but somewhat involved expressions for multinomial coefficients, ultimately obtaining the following result. For any integer $k \geq 1$, there is a polynomial $P_{k}(x)$ of degree $k$ with $P_{k}(0)=0$ such that

$$
\int_{0}^{t_{0}} e^{-d t}\left(\left(\sum_{n=0}^{N-1} a_{n} t^{2 n}\right)^{d}-1\right) \frac{d t}{t}=P_{k}(1 / d)+O\left(d^{-k-1}\right), \quad \text { as } d \rightarrow \infty .
$$

We leave the details of these computations to the interested reader.

\subsection{The spectral zeta function}

In very general circumstances, the spectral zeta function is defined as the Mellin transform of the theta function formed with the nonzero eigenvalues. Specifically, for $w \in \mathbf{C}$ with $\operatorname{Re}(w)>d / 2$, we have that

$$
\zeta_{N}(w)=\mathbf{M} \theta_{N}(w)=\frac{1}{\Gamma(w)} \int_{0}^{\infty}\left(\theta_{N}(t)-1\right) t^{w} \frac{d t}{t} .
$$

As in Section 5, let us consider a degenerating sequence of discrete tori. The elementary change of variables $t \mapsto u^{2} t$ in (74) yields the expression

$$
\zeta_{N}(w)=\frac{u^{2 w}}{\Gamma(w)} \int_{0}^{\infty}\left(\theta_{N}\left(u^{2} t\right)-1\right) t^{w} \frac{d t}{t}
$$

Proposition 5.2 establishes the pointwise convergence of the integrand in (75), and Lemma 5.3 proves a uniform upper bound. Combining these results, as in the proof of Proposition 5.4, together with (75), we conclude that

$$
\lim _{u \rightarrow \infty}\left(u^{-2 w} \zeta_{N(u)}(w)\right)=\zeta_{A}(w)
$$

for any $w \in \mathbf{C}$ with $\operatorname{Re}(w)>d / 2$ and where

$$
\zeta_{A}(w)=\frac{1}{\Gamma(w)} \int_{0}^{\infty}\left(\Theta_{A}(t)-1\right) t^{w} \frac{d t}{t}
$$


is the spectral zeta function on the real torus $A \mathbf{Z}^{d} \backslash \mathbf{Z}^{d}$. The difficulty is determining the correct generalization of $(76)$ for $w \in \mathbf{C}$ with $\operatorname{Re}(w)>0$. The spectral zeta function (75) can be meromorphically continued to $w \in \mathbf{C}$ with $\operatorname{Re}(w)>0$ by writing

$$
\begin{aligned}
u^{-2 w} \zeta_{N}(w)= & \frac{1}{\Gamma(w)} \int_{1}^{\infty}\left(\theta_{N}\left(u^{2} t\right)-1\right) t^{w} \frac{d t}{t} \\
& +\frac{1}{\Gamma(w)} \int_{0}^{1}\left(\theta_{N(u)}\left(u^{2} t\right)-V(N) e^{-2 d u^{2} t} I_{0}\left(2 u^{2} t\right)^{d}\right) t^{w} \frac{d t}{t} \\
& -\frac{1}{\Gamma(w)} \int_{0}^{1}\left(V(N) e^{-2 d u^{2} t} I_{0}\left(2 u^{2} t\right)^{d}-1\right) t^{w} \frac{d t}{t}
\end{aligned}
$$

Similarly, the meromorphic continuation of (77) is obtained by the expression

$$
\begin{aligned}
\zeta_{A}(w)= & \frac{1}{\Gamma(w)} \int_{1}^{\infty}\left(\Theta_{A}(t)-1\right) t^{w} \frac{d t}{t} \\
& +\frac{1}{\Gamma(w)} \int_{0}^{1}\left(\Theta_{A}(t)-V(A)(4 \pi t)^{-d / 2}\right) t^{w} \frac{d t}{t} \\
& -\frac{1}{\Gamma(w)} \int_{0}^{1}\left(V(A)(4 \pi t)^{-d / 2}-1\right) t^{w} \frac{d t}{t}
\end{aligned}
$$

The convergence of the first integral in (78) to the first integral in (79) follows from Proposition 5.2 and Lemma 5.3, as in the proof of (76). The convergence of the second integral in (78) to the second integral in (79) follows from Proposition 5.5, using the Lebesgue dominated convergence theorem. Let us write the third integral in (78) as

$$
\begin{aligned}
& \int_{0}^{1}\left(V(N) e^{-2 d u^{2} t} I_{0}\left(2 u^{2} t\right)^{d}-1\right) t^{w} \frac{d t}{t} \\
& =\frac{V(N)}{u^{2 w}} \int_{0}^{u^{2}}\left(e^{-2 t} I_{0}(2 t)\right)^{d} t^{w} \frac{d t}{t}-\frac{1}{w}
\end{aligned}
$$

and the third integral in (77) as

$$
\int_{0}^{1}\left(V(A)(4 \pi t)^{-d / 2}-1\right) t^{w} \frac{d t}{t}=\frac{V(A)}{(4 \pi)^{d / 2}(w-d / 2)}-\frac{1}{w} .
$$


Combining all of these results, we have shown that

$$
\begin{gathered}
\lim _{u \rightarrow \infty} u^{-2 w}\left(\zeta_{N}(w)-\frac{V(N)}{\Gamma(w)} \int_{0}^{u^{2}}\left(e^{-2 t} I_{0}(2 t)\right)^{d} t^{w} \frac{d t}{t}\right) \\
=\zeta_{A}(w)-\frac{V(A)}{(4 \pi)^{d / 2}(w-d / 2) \Gamma(w)} .
\end{gathered}
$$

Using Proposition 4.7, one can easily show that (82) is equivalent to (76) when $\operatorname{Re}(w)>d / 2$, noting that $(82)$ holds for all $w \in \mathbf{C}$ with $\operatorname{Re}(w)>0$ provided $\operatorname{Re}(w) \neq d / 2$. By continuity, (82) extends to all $w \in \mathbf{C}$ with $\operatorname{Re}(w)>0$ provided one uses the interpretation

$$
\lim _{w \rightarrow d / 2}\left(\zeta_{A}(w)-\frac{V(A)}{(4 \pi)^{d / 2}(w-d / 2) \Gamma(w)}\right)=\mathrm{CT}_{w=d / 2} \zeta_{A}(w),
$$

the constant term in the Laurent expansion at $w=d / 2$.

The above computations in the special case $d=2$ with $w=1$ and $w=2$ were obtained in [9, Section 3]. In the case when $w=1$ and $d=2$, so then $w=d / 2$, the results in [9] were expressed in terms of the modular forms. However, in light of the general Kronecker limit formula for Epstein zeta functions and its functional equation, as stated in Section 2.9, it is evident that for general $d \neq 1$, the limiting value obtained in (83) can be expressed in terms of a modular form (22).

\subsection{The Epstein-Hurwitz zeta function}

Further analysis in [9] involves the investigation of the finite product $\prod\left(s^{2}+\Lambda_{j}\right)$ for degenerating families of two-dimensional discrete tori for general $s \in \mathbf{C}$. If $s=0$, then Theorem 5.8 determines the asymptotic behavior of the product of nonzero eigenvalues. For $s \neq 0$, we include the zero eigenvalues, which trivially introduces the multiplicative factor of $s^{2}$. In this setting, we recall that Theorem 3.6 establishes a relation for $\prod\left(s^{2}+\Lambda_{j}\right)$ in terms of integrals involving $I$-Bessel functions. Using the substitution $t \mapsto u^{2} t$, we arrive at the expression

$$
\begin{aligned}
\log \prod\left((s / u)^{2}+\Lambda_{j}\right) & =\sum_{\Lambda_{j} \neq 0} \log \left((s / u)^{2}+\Lambda_{j}\right) \\
& =V(N / u) \mathcal{I}_{d}(s / u)+\mathcal{H}_{N}(s / u),
\end{aligned}
$$

where

$$
\mathcal{I}_{d}(s / u)=-\int_{0}^{\infty}\left(e^{-2 d u^{2} t} e^{-s^{2} t} I_{0}\left(2 u^{2} t\right)^{d}-e^{-u^{2} t}\right) \frac{d t}{t}
$$


and

$$
\mathcal{H}_{N}(s / u)=-\int_{0}^{\infty} e^{-s^{2} t}\left(\theta_{N}\left(u^{2} t\right)-V(N) e^{-2 d u^{2} t} I_{0}\left(2 u^{2} t\right)^{d}\right) \frac{d t}{t}-\log \left(s^{2}\right)
$$

Trivially, one has that

$$
\prod\left((s / u)^{2}+\Lambda_{j}\right)=u^{-2 V(N)} \prod\left(s^{2}+u^{2} \cdot \Lambda_{j}\right)
$$

We can now employ bounds from Section 5 in order to determine the asymptotic behavior of (84) as $u \rightarrow \infty$. Specifically, one uses Propositions 4.7, 5.2, and 5.5 and Lemma 5.3 to show that for any $s \in \mathbf{C}$ with $\operatorname{Re}\left(s^{2}\right)>0$ we have the asymptotic formula

$$
\prod\left((s / u)^{2}+\Lambda_{j}\right)=V(A / u) \mathcal{I}_{d}(s / u)+\mathcal{H}_{A}(s)+o(1), \quad \text { as } u \rightarrow \infty,
$$

where

$$
\mathcal{H}_{A}(s)=-\int_{0}^{\infty} e^{-s^{2} t}\left(\Theta_{A}(t)-V(A)(4 \pi t)^{-d / 2}\right) \frac{d t}{t}-\log \left(s^{2}\right)
$$

The function $\mathcal{H}_{A}(s)$ is related to the regularized harmonic series, as defined and studied in [13] associated to the set $\left\{\Lambda_{A, j}+s^{2}\right\}$ where $\left\{\Lambda_{A, j}\right\}$ is the set of eigenvalues of the Laplacian on the real torus $A \mathbf{Z}^{d} \backslash \mathbf{R}^{d}$. The general results from [13] establish that $\mathcal{H}_{A}(s)$ can be expressed as special values of the Epstein-Hurwitz zeta function formed with the set $\left\{\Lambda_{A, j}+s^{2}\right\}$. We refer the reader to [13] for further details and identities.

\subsection{General discrete tori}

Let $B$ be a positive definite $d \times d$ integer matrix, and consider the discrete tori $D T_{B}=B \mathbf{Z}^{d} \backslash \mathbf{Z}^{d}$. The results from [15] easily extend to compute the spectrum of the Laplacian on $D T_{B}$ in terms of the dual lattice $B^{*}$. The existence and uniqueness of the associated heat kernel on $D T_{B}$ follow from general results (see, e.g., [6], [7]), thus allowing one to extend the results of Section 3 above. Assume there is a one-parameter family $D T_{B(u)}$ of discrete tori parametrized by $u \in \mathbf{Z}$ such that $B(u) / u \rightarrow M$ as $u \rightarrow \infty$, where $M$ is a positive definite $d \times d$ matrix. The results of the present article apply when considering spectral invariants on $D T_{B(u)}$. For example, Theorem 5.8 will extend to obtain the asymptotic behavior of the determinant of the Laplacian on $D T_{B(u)}$ with second-order term equal to the zeta-regularized spectral determinant on the real torus $M \mathbf{Z}^{d} \backslash \mathbf{R}^{d}$. Additional results of the present article, specifically the contents of Sections 7.3 and 7.4, carry through using the proofs given and only a slight change in notation. 


\subsection{Height functions}

The asymptotic expansion from Theorem 5.8, and its extension as outlined in Section 7.5 , can be interpreted as saying that $\log \operatorname{det}^{*} \boldsymbol{\Delta}_{D T, *}$ is a height function on the space of discrete tori of fixed dimension since it tends to $+\infty$ through degeneration. In the case of real tori, one needs to introduce a minus sign and study $-\log \operatorname{det}^{*} \boldsymbol{\Delta}_{\mathrm{RT}, *}$ in order to have a height function which tends to $+\infty$ through degeneration (see, e.g., [5]). The problem of finding real tori with minimum height remains a question of interest (see, e.g., [23]). One point of future investigation is to see to what extent Theorem 5.8 allows for a connection between the problems considered in [23] and the study of the height function $\log \operatorname{det}^{*} \boldsymbol{\Delta}_{D T, *}$ on discrete tori, which has the advantage of being an invariant which is defined as a finite product and not through meromorphic continuation.

Acknowledgments. We are grateful to the Hausdorff Center in Bonn for the opportunity for us to meet in the summer of 2007.

\section{REFERENCES}

[1] K. Athreya, Modified Bessel function asymptotics via probability, Statist. Probab. Lett. 5 (1987), 325-327.

[2] R. Burton and R. Pemantle, Local characteristics, entropy and limit theorems for spanning trees and domino tilings via transfer-impedances, Ann. Prob. 21 (1993), $1329-1371$.

[3] M. Casartelli, L. Dall'Asta, A. Vezzani, and P. Vivo, Dynamical invariants in the deterministic fixed-energy sandpile, Eur. Phys. J. B 52 (2006), 91-105.

[4] S.-C. Chang and R. Shrock, Some exact results for spanning trees on lattices, J. Phys. A 39 (2006), 5653-5658.

[5] P. Chiu, Height of flat tori, Proc. Amer. Math. Soc. 125 (1997), 723-730.

[6] J. Dodziuk, "Elliptic operators on infinite graphs," in Krzysztof Wojciechowski 50 Years-Analysis and Geometry of Boundary Value Problems, World Scientific, Hackensack, NJ, 2006, 353-368.

[7] J. Dodziuk and V. Mathai, "Kato's inequality and asymptotic spectral properties for discrete magnetic Laplacians," in The Ubiquitous Heat Kernel, Contemp. Math. 398, Amer. Math. Soc., Providence, 2006, 269-297.

[8] W. Duke and Ö. Imamog̈lu, Special values of multiple gamma functions, J. Théor. Nombres Bordeaux 18 (2006), 113-123.

[9] B. Duplantier and F. David, Exact partition functions and correlation functions of multiple Hamiltonian walks on the Manhattan lattice, J. Stat. Phys. 51 (1988), 327434.

[10] P. Epstein, Zur Theorie allgemeiner Zetafunctionen, Math. Ann. 56 (1903), 615-644.

[11] J. Felker and R. Lyons, High-precision entropy values for spanning trees in lattices, J. Phys. A 36 (2003), 8361-8365.

[12] C. Godsil and G. Royce, Algebraic Graph Theory, Grad. Texts Math. 207, Springer, Berlin, 2001. 
[13] J. Jorgenson and S. Lang, "Complex analytic properties of regularized products and series," in Basic Analysis of Regularized Products and Series, Lecture Notes in Math. 1564, Springer, Berlin, 1993.

[14] J. Jorgenson and S. Lang, "The ubiquitous heat kernel," in The Mathematics Unlimited - 2001 and Beyond, Springer, Berlin, 2001, 655-683.

[15] A. Karlsson and M. Neuhauser, "Heat kernels, theta identities, and zeta functions on cyclic groups," in Topological and Asymptotic Aspects of Group Theory, Contemp. Math. 394, Amer. Math. Soc., Providence, 2006, 177-189.

[16] P. W. Kasteleyn, The statistics of dimers on a lattice, I, The number of dimer arrangements on a quadratic lattice, Physica 27 (1961), 1209-1225.

[17] R. Kenyon, The asymptotic determinant of the discrete Laplacian, Acta Math. 185 (2000), 239-286.

[18] G. Kirchhoff, Über die Auflösung der Gleichungen, auf welche man bei der Untersuchung der linearen Verteilung galvanischer Strome gefurt wird, Ann. Phys. Chem. 72 (1847), 497-508.

[19] B. Osgood, R. Phillips, and P. Sarnak, Extremals of determinants of Laplacians, J. Funct. Anal. 80 (1988), 148-211.

[20] C. O'Sullivan, Formulas for non-holomorphic Eisenstein series, in preparation.

[21] B. V. Pal'tsev, Two-sided bounds uniform in the real argument and the index for modified Bessel functions, Math. Notes 65 (1999), 571-581.

[22] P. Sarnak, "Determinants of Laplacians; heights and finiteness," in Analysis, et cetera, Academic Press, Boston, 1990, 601-622.

[23] P. Sarnak and A. Strombergsson, Minima of Epstein's zeta function and heights of flat tori, Invent. Math. 165 (2006), 115-151.

[24] R. Shrock and F. Y. Wu, Spanning trees on graphs and lattices in d dimensions, J. Phys. A 33 (2000), 3881-3902.

[25] A. Sokal and A. Starinets, Pathologies of the large- $N$ limit for $\boldsymbol{R} P^{N}-1, \boldsymbol{C} P^{N-1}$, $Q P^{N-1}$ and mixed isovector/isotensor $\sigma$-models, Nuclear Phys. B 601 (2001), 425502 .

Gautam Chinta

Department of Mathematics

The City College of New York

Convent Avenue at 138th Street

New York, New York 10031

USA

chinta@sci.ccny. cuny. edu

Jay Jorgenson

Department of Mathematics

The City College of New York

Convent Avenue at 138th Street

New York, New York 10031

$U S A$

jjorgenson@mindspring.com 
Anders Karlsson

Mathematics Department

Royal Institute of Technology

10044 Stockholm

Sweden

akarl@kth.se 